\begin{tabular}{lc} 
Sharif University of Technology \\
Scientia Iranica \\
SCIENTIA & Transactions B: Mechanical Engineering \\
IRAN ICA & www.scientiairanica.com \\
\hline
\end{tabular}

\title{
Effect of alternating magnetic field on unsteady MHD mixed convection and entropy generation of ferrofluid in a linearly heated two-sided cavity
}

\author{
O. Ghaffarpasand* \\ Department of Physics, University of Isfahan, Isfahan, 81746, Iran.
}

Received 20 October 2015; received in revised form 7 May 2016; accepted 28 June 2016

KEYWORDS
MHD mixed
convection;
Ferrofluid;
Two-sided cavity;
Linearly heating;
Time-periodic
magnetic field.

\section{Introduction}

The problem on mixed convection heat transfer in cavities with moving wall(s) has multiple applications in practical situations and natural phenomena such as lakes and large reservoirs, nuclear reactors, highperformance building insulation, lubrication technologies, solar collectors, cooling of microprocessors, and crystal growth [1,2]. Traditional fluids, utilized for heat transfer applications, such as water, mineral oils, and ethylene glycol, usually have a rather low thermal conductivity; therefore, new techniques have emerged to improve their thermal conductivity. One of them is adding a minor volume fraction of nano-scale particles

\footnotetext{
*. Tel.: +98 $3137934843 ;$ Fax: +983137934800

E-mail address: o.ghaffarpasand@gmail.com
}

\begin{abstract}
This work considers the numerical study of unsteady MHD convection of flow characteristics and heat transfer. The entropy generation and Bejan number are also has remarkably influenced the fluid characteristics, the fluid irreversibilities, and heat seems to be insignificant.
\end{abstract}

(C) 2017 Sharif University of Technology. All rights reserved. of size up 1-100 $\mathrm{nm}$ with high thermal conductivity in the base fluid $[3,4]$. The product is called a nanofluids by Choi [5].

Of particular interest to researchers nowadays is ferrofluid, where stable colloidal suspensions of subdomain magnetic particles are utilized as nanoparticles in nanofluids [6,7]. $\mathrm{Fe}_{3} \mathrm{O}_{4}$, cobalt, and nickel nanoparticles are commonly used for this purpose and cobalt-kerosene ferrofluid has better heat transfer characteristics than the others. This ferrofluid is less costly and environmentally friendly [8]. However, one of the popular techniques to control convective flow fields of fluid systems containing nanofluid or ferrofluid is applying an external magnetic field. Magnetohydrodynamic (MHD) convection of nanofluids/ferrofluids has received much attention during the recent years due to its importance in micro-electronic devices, cooling 
of nuclear reactors, purification of molten metals, design of MHD pumps and accelerators, and many others [7]. Rahman et al. utilized statistical and numerical techniques to study unsteady MHD convection of cobalt-kerosene ferrofluid in a semicircularshaped enclosure [8]. They found that the higher solid volume fraction of ferroparticles in a base fluid sufficiently improved its thermo-physical properties. Mohamadpourfard numerically examined the hydrothermal behavior of ferrofluid in a rectangular minichannel in the presence of a space-dependent nonuniform magnetic field [9]. The results showed that the convective heat transfer was improved by applying a magnetic field with a negative gradient. Sheikholeslami \& Ganji numerically studied MHD convection of ferrofluid in a semicircular annulus and concluded that the increments of solid volume fraction of ferrofluid along with Rayleigh number increasing played a positive role in augmentation of heat transfer [10]. Selimefendigil et al. numerically investigated mixed convection of $\mathrm{Cu}$ water nanofluid filled lied-driven cavity when different inclined magnetic fields were applied to upper and lower triangular domains [11]. Their results indicated that the local and averaged heat transfer were deteriorated with increase of Richardson number. Ahrar et al. utilized the Lattice Boltzmann Method (LBM) to study the influence of volume fraction and magnetic field specification on flow and heat transfer of $\mathrm{Cu}$ water nanofluid inside an enclosed enclosure [12]. It was shown that the influence of magnetic field direction on heat transfer was pronounced in moderate or high Rayleigh numbers. Rashidi et al. numerically examined the influence of magnetic field on mixed convection heat transfer of nanofluid in a channel with sinusoidal walls [13]. The results also showed that the average Nusselt number increased with enhancement of Grashof number for nanofluids with different volume fractions. Garoosi \& Hosseininejad performed numerical simulations to study mixed convection between differentially heated cylinders in an adiabatic enclosure filled with nanofluid [14]. The results indicated that the place of heated cylinders was an important factor in the heat transfer variation within the enclosure.

It is worthwhile to note that Maxwell-Garnett's (MG) model is the commonly used model in the literature to estimate the effective thermal conductivity of nanofluids/ferrofluids. The MG model is a special case of Hamilton-Crosser model where the shape of nanoparticles is assumed to be spherical and depends on thermal conductivity of the base fluid and nanofluid as well as the nanoparticle volume fraction [15]. Besides, the Brinkman model was widely utilized to examine the effective nanofluid/ferrofluid viscosity [16] when it was assumed that the suspension of spherical nanoparticles was dilute enough.

The magnetization of ferrofluid is strongly de- pendent on the external magnetic field, whereby any temporal or spatial variation induces change of fluid characteristics and heat transfer. Nevertheless, in almost the whole previous studies, the applied external magnetic field has a certain value during the simulation and the influence of temporal variation of magnetic field on fluid characteristics and heat transfer of ferrofluid flow has not been well researched yet. Recently, Sadrhosseini et al. experimentally investigated the effect of time-dependent magnetic field on the ferrofluid flow inside a tube [17]. They just observed that an appropriate magnetic field mode accompanied with the proper frequency could make an enhancement in convective heat transfer.

In any physical process, some amount of available energy is wasted during the course of processes, e.g. viscous friction, thermal gradient, external magnetic field, or even chemical reactions. Entropy generation is an important factor in the energy of thermal systems; its minimization has been considered as an optimal design criterion [18]. Entropy Generation Minimization (EGM) was firstly studied by Bejan [19]. He discussed the concept of entropy and proved that temperature gradient, along with the viscous effects, is the main factor contributing to the EGM. During the past two decades, research based on the entropy generation analysis has also emerged [2]. Nevertheless, although some studies on MHD convection of ferrofluids are available, the corresponding research analyzing entropy generation is quite sparse. Nayak et al. numerically studied the entropy generation in mixed convection of copper-water nanofluid inside a differentially heated skewed cavity [18]. They observed that heat transfer and entropy generation were enough influenced by skew angle variation. Mejri et al. employed the LBM to investigate entropy generation in a nanofluid filled enclosure with sinusoidal heating on both side walls [20]. They found that Hartmann and solid volume fraction were important factors affecting entropy generation inside the cavity. On the other hand, most of the existing studies have been performed in the stationary or open cavities and have not considered the role of mechanical forces induced by moving lid(s) in the fluid irreversibilities and entropy generation.

From the above literature survey, it is evident that although significant studies have been reported for heat transfer enhancement of nanofluid in the presence of constant magnetic field, to the author's best knowledge, the problem of unsteady MHD convection of ferrofluids in the presence of an alternating/timedependent magnetic field has not been well analyzed. Moreover, entropy generation for the mentioned problem is the other issue, which has not been investigated yet. Therefore, the main purpose of this study is to characterize heat transfer and fluid flow of MHD convection of ferrofluid in the presence of a time- 
Table 1. Thermo-physical properties of Cobalt-Kerosene ferrofluid [8].

\begin{tabular}{cccccc}
\hline $\begin{array}{c}\text { Physical } \\
\text { properties }\end{array}$ & $\begin{array}{c}\boldsymbol{\rho} \\
\left(\mathbf{k g m}^{-\mathbf{3}}\right)\end{array}$ & $\begin{array}{c}\boldsymbol{C}_{\boldsymbol{p}} \\
\left(\mathbf{J k g}^{-\mathbf{1}} \mathbf{K}^{-\mathbf{1}}\right)\end{array}$ & $\begin{array}{c}\boldsymbol{k} \\
\left(\mathbf{W m}^{-\mathbf{1}} \mathbf{K}^{-\mathbf{1}}\right)\end{array}$ & $\begin{array}{c}\boldsymbol{\beta} \times \mathbf{1 0}^{-\mathbf{5}} \\
\left(\mathbf{K}^{-\mathbf{1}}\right)\end{array}$ & $\begin{array}{c}\boldsymbol{\sigma} \\
\left(\mathbf{S m}^{-\mathbf{1}}\right)\end{array}$ \\
\hline Kerosene & 780 & 2090 & 0.149 & 99 & $6 \times 10^{-10}$ \\
Cobalt & 8900 & 420 & 100 & 1.3 & $1.6 \times 10^{7}$ \\
\hline
\end{tabular}

periodic external magnetic field. In addition, the factors which control entropy generation are analyzed and discussed. Cobalt-kerosene ferrofluid is used in this study due to its specific properties mentioned earlier. It is worthwhile to note that two-sided cavity with linear heating of the bottom wall is also utilized to make a unique opportunity to better discuss the role of mechanical forces in addition to thermal buoyancy forces on heat transfer and fluid irreversibilities of MHD mixed convection of ferrofluids. This combination has not been yet utilized in the literature.

\section{Problem definition and mathematical formulation}

The geometry and coordinate system of the physical model are displayed in Figure 1. It consists of a ferrofluid filled two-sided cavity with unity aspect ratio $(L=1)$, in the presence of a constant and alternating/time-periodic external magnetic field. Vertical walls move with constant velocity in the same direction and are adiabatic and impermeable. The horizontal top wall is kept at constant temperature equal to minimum temperature of linear heating on the bottom wall. The applied external magnetic field is in the $x$ direction. The fluid inside the cavity is a Newtonian and incompressible kerosene based ferrofluid containing cobalt ferroparticles. In addition, the base fluid and suspended nanoparticles are assumed

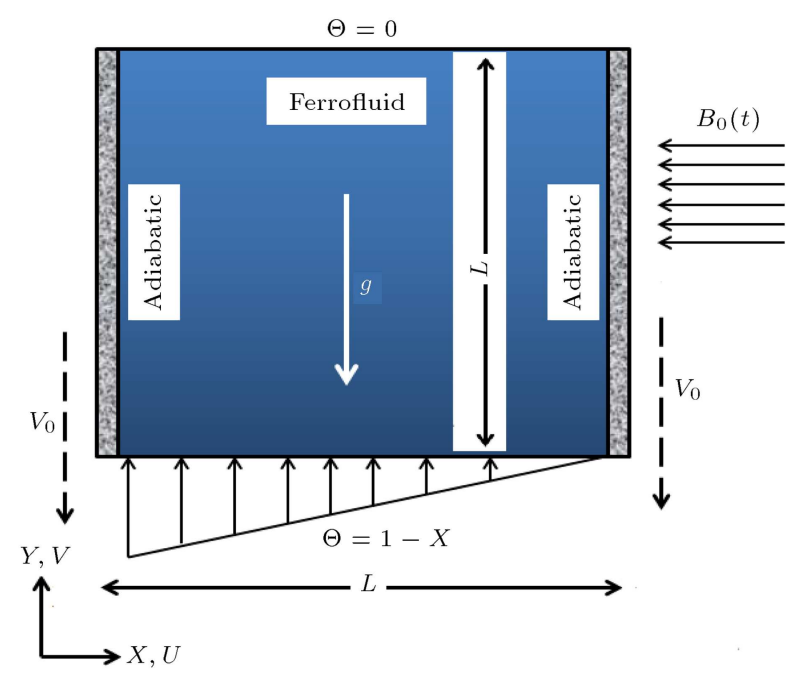

Figure 1. Physical system and Cartesian coordinates, where $L=1$. Dashed arrows represent the direction of moving walls. in thermal equilibrium without slip occurring between the two phases. Thermo-physical properties of the ferrofluid are listed in Table 1.

A first-order Boussinesq approximation is used in this study; thus, thermo-physical properties of ferrofluid are assumed to be constant except for density in the buoyancy force term. Based on the model, the governing dimensional equations for the unsteady, two-dimensional, laminar, and incompressible mixed convection, which include continuity, Navier-Stokes, and energy equations, are expressed as:

$$
\begin{aligned}
& \frac{\partial u}{\partial x}+\frac{\partial v}{\partial y}=0 \\
& \frac{\partial u}{\partial t}+\frac{\partial(u u)}{\partial x}+\frac{\partial(v u)}{\partial y}=-\frac{1}{\rho_{f f}} \frac{\partial p}{\partial x} \\
& \quad+\frac{\mu_{f f}}{\rho_{f f}}\left(\frac{\partial^{2} u}{\partial x^{2}}+\frac{\partial^{2} u}{\partial y^{2}}\right) \\
& \frac{\partial v}{\partial t}+\frac{\partial(u v)}{\partial x}+\frac{\partial(v v)}{\partial y}=-\frac{1}{\rho_{f f}} \frac{\partial p}{\partial y} \\
& \quad+\frac{\mu_{f f}}{\rho_{f f}}\left(\frac{\partial^{2} v}{\partial x^{2}}+\frac{\partial^{2} v}{\partial y^{2}}\right)+\frac{(\rho \beta)_{f f}}{\rho_{f f}} g\left(T-T_{c}\right) \\
& \quad+\frac{\sigma_{f f} B_{0}^{2}}{\rho_{f f}} v
\end{aligned}
$$$$
\frac{\partial T}{\partial t}+\frac{\partial(u T)}{\partial x}+\frac{\partial(v T)}{\partial y}=\alpha_{f f}\left(\frac{\partial^{2} T}{\partial x^{2}}+\frac{\partial^{2} T}{\partial y^{2}}\right),
$$

where the effective density at the reference temperature, thermal diffusivity, heat capacitance, thermal expansion coefficient, and conductivity of the ferrofluid are respectively written as follows [8]:

$$
\begin{aligned}
& \rho_{f f}=(1-\phi) \rho_{f}+\phi \rho_{s}, \\
& \alpha_{f f}=\frac{k_{f f}}{\left(\rho C_{p}\right)_{f f}}, \\
& \left(\rho C_{p}\right)_{f f}=(1-\phi)\left(\rho C_{p}\right)_{f}+\phi\left(\rho C_{P}\right)_{s}, \\
& (\rho \beta)_{f f}=(1-\phi)(\rho \beta)_{f}+\phi(\rho \beta)_{s}, \\
& \sigma_{f f}=\sigma_{f}\left(1+\frac{3\left(\frac{\sigma_{s}}{\sigma_{f}}-1\right) \phi}{\left(\frac{\sigma_{s}}{\sigma_{f}}+2\right)-\left(\frac{\sigma_{s}}{\sigma_{f}}-1\right) \phi}\right) .
\end{aligned}
$$


Here, $\phi$ is the solid volume fraction of the nanoparticles. It is important to note that two kinds of external magnetic fields are used in this study: constant and time-periodic, which is expressed as:

$$
B(t)=B_{0} \sin (\omega t+\varphi) .
$$

$\omega=\frac{2 \pi}{t_{B}}$ and $\varphi$ are angular frequency and phase deviation, respectively, where $t_{B}$ is the period of timedependent magnetic field. The magnetic Reynolds number, $\operatorname{Re}_{m}$, is assumed to be small enough $\left(\operatorname{Re}_{m}=\right.$ $\mu_{f} \sigma_{f} V_{0} L \ll 1$ ), which enables us to ignore the induced magnetic field compared to the applied magnetic field. The primary benefit of this hypothesis, which is known as the low-Rem approximation, is a significant reduction of the number of equations required to be solved. The limits of validity of the low-Rem approximation were examined numerically by Sarris et al. [21]. They evaluated the errors associated with that approximation in MHD natural convection and found that the differences were negligible at large values of Grashof numbers, moderate values of Hartmann number, and large values of Prandtl numbers. Besides, the influence of induced magnetic field on ferrite nanoparticles and vis versa could be neglected with respect to the small value of assumed magnetic Reynolds number. A similar trend was utilized before by Sheikholeslami \& Ganji [10] and Rahman et al. [8]. However, there is no electric field applied to the flow. It should be noted that the magnetization coefficient for a particle size of $45 \mathrm{~nm}$ is equal to unity [2]. The Maxwell-Garnett's (MG) model is utilized in this study to examine the thermal conductivity of the ferrofluid. Hence, for twocomponent velocity of spherical-particle suspension, effective thermal conductivity of the ferrofluid is written by:

$$
\frac{k_{f f}}{k_{f}}=\frac{k_{s}+2 k_{f}-2 \phi\left(k_{f}-k_{s}\right)}{k_{s}+2 k_{f}+\phi\left(k_{f}-k_{s}\right)} .
$$

Brinkman model is also used here to examine the effective ferrofluid viscosity as follows:

$$
\mu_{f f}=\frac{\mu_{f}}{(1-\phi)^{2.5}} .
$$

The governing equations are non-dimensionalized using the following dimensionless variables:

$$
\begin{array}{ll}
(X, Y)=\frac{(x, y)}{L}, & (U, V)=\frac{(u, v)}{V_{0}}, \\
\Theta=\frac{T-T_{c}}{T_{h}-T_{c}}, \quad \tau=\frac{t V_{0}}{L}, \quad P=\frac{p}{\rho_{f f} V_{0}^{2}} .
\end{array}
$$

Then, the dimensionless form of governing equations become:

$$
\frac{\partial U}{\partial X}+\frac{\partial V}{\partial Y}=0
$$

$$
\begin{aligned}
\frac{\partial U}{\partial \tau} & +\frac{\partial(U U)}{\partial X}+\frac{\partial(V U)}{\partial Y}=-\frac{\partial P}{\partial X} \\
& +\frac{\rho_{f}}{\rho_{f f}} \frac{1}{(1-\phi)^{2.5}} \frac{1}{\operatorname{Re}}\left(\frac{\partial^{2} U}{\partial X^{2}}+\frac{\partial^{2} U}{\partial Y^{2}}\right) \\
\frac{\partial V}{\partial \tau} & +\frac{\partial(U V)}{\partial X}+\frac{\partial(V V)}{\partial Y}=-\frac{\partial P}{\partial Y} \\
& +\frac{\rho_{f}}{\rho_{f f}} \frac{1}{(1-\phi)^{2.5}} \frac{1}{\operatorname{Re}}\left(\frac{\partial^{2} V}{\partial X^{2}}+\frac{\partial^{2} V}{\partial Y^{2}}\right) \\
& +\operatorname{Ri} \frac{\rho_{f}}{\rho_{f f}}\left(1-\phi+\frac{\rho_{s} \beta_{s}}{\rho_{f} \beta_{f}} \phi\right) \Theta \\
& +\frac{1}{\operatorname{Re}} \frac{\rho_{f}}{\rho_{f f}} \frac{\sigma_{f f}}{\sigma_{f}} \mathrm{Ha}^{2} V \\
\frac{\partial \Theta}{\partial \tau}+ & \frac{\partial(U \Theta)}{\partial X}+\frac{\partial(V \Theta)}{\partial Y}=\frac{\alpha_{f f}}{\alpha_{f}} \frac{1}{\operatorname{RePr}}\left(\frac{\partial^{2} \Theta}{\partial X^{2}}+\frac{\partial^{2} \Theta}{\partial Y^{2}}\right)
\end{aligned}
$$

where Reynolds, Richardson, Grashof, Prandtl, and Hartmann numbers are respectively defined as:

$$
\begin{array}{ll}
\operatorname{Re}=\frac{V_{0} L}{\nu_{f}}, & \mathrm{Ri}=\frac{\mathrm{Gr}}{\operatorname{Re}^{2}}, \quad \mathrm{Gr}=\frac{g \beta_{f} \Delta T L^{3}}{\nu_{f}^{3}}, \\
\operatorname{Pr}=\frac{\nu_{f}}{\alpha_{f}}, & \mathrm{Ha}=B_{0} \sqrt{\frac{\sigma_{f} L}{\mu_{f}}} .
\end{array}
$$

The dimensionless boundary conditions associated with the problem are as follows:

$$
\begin{gathered}
U=0, \quad V=-1, \quad \frac{\partial \Theta}{\partial X}=0 \\
\text { for } \quad X=0, \quad \text { and } \quad 0 \leq Y \leq 1, \\
U=0, \quad V=-1, \quad \frac{\partial \Theta}{\partial X}=0 \\
\text { for } \quad X=1, \quad \text { and } \quad 0 \leq Y \leq 1, \\
U=V=0, \quad \Theta=1-X \\
\text { for } \quad 0 \leq X \leq 1, \quad \text { and } \quad Y=0, \\
U=V=0, \quad \Theta=0 \\
\text { for } \quad 0 \leq X \leq 1, \quad \text { and } Y=1 .
\end{gathered}
$$

To examine the overall heat transfer across the cavity, the local and average Nusselt numbers are calculated along the heated bottom wall. The local Nusselt number, $\mathrm{Nu}$, can be expressed as:

$$
\mathrm{Nu}(X)=\frac{h_{f f} L}{k_{f}},
$$


where the heat transfer coefficient of ferrofluid, $h_{f f}$, is given by:

$$
h_{f f}=-\left.\frac{k_{f f}}{L} \frac{\partial \Theta}{\partial Y}\right|_{Y=0} .
$$

Therefore, the local Nusselt number, and further the average Nusselt number, $\overline{\mathrm{Nu}}$, can be written as:

$$
\mathrm{Nu}(X)=-\left.\frac{k_{f f}}{k_{f}} \frac{\partial \Theta}{\partial Y}\right|_{Y=0}, \quad \overline{\mathrm{Nu}}=\int_{0}^{1} \mathrm{Nu}(X) d X
$$

In the studied problem, entropy generation is due to irreversibilities generated through temperature gradient, fluid friction, and magnetic field. As a result, local entropy generation is the sum of irreversibilities due to viscous dissipation, thermal gradients, and magnetic field as follows:

$$
S=S_{F}+S_{T}+S_{B},
$$

where:

$$
\begin{aligned}
& S_{F}=\frac{\mu_{f f}}{T_{m}}\left[2\left(\frac{\partial u}{\partial x}\right)^{2}+2\left(\frac{\partial v}{\partial y}\right)^{2}+\left(\frac{\partial u}{\partial y}+\frac{\partial v}{\partial x}\right)^{2}\right] \\
& S_{T}=\frac{k_{f f}}{T_{m}^{2}}\left[\left(\frac{\partial T}{\partial x}\right)^{2}+\left(\frac{\partial T}{\partial y}\right)^{2}\right] \\
& S_{B}=\frac{\sigma_{f f} B_{0}^{2} v^{2}}{T_{m}}
\end{aligned}
$$

Here, $T_{m}=\left(T_{h}+T_{c}\right) / 2$ is the reference temperature. $S_{F}, S_{B}$, and $S_{T}$ are called viscous dissipation, magnetic, and thermal entropy generation, respectively, hereinafter. By using dimensionless parameters, total entropy in dimensionless form can be written as:

$$
\begin{aligned}
S^{\prime}= & S_{F}^{\prime}+S_{T}^{\prime}+S_{B}^{\prime}=S \frac{T_{m} L^{2}}{k_{f}\left(T_{h}-T_{c}\right)^{2}} \\
= & \chi \frac{\mu_{f f}}{\mu_{f}}\left[2\left(\frac{\partial U}{\partial X}\right)^{2}+2\left(\frac{\partial V}{\partial Y}\right)^{2}+\left(\frac{\partial U}{\partial Y}+\frac{\partial V}{\partial X}\right)^{2}\right] \\
& +\frac{k_{f f}}{k_{f}}\left[\left(\frac{\partial \Theta}{\partial X}\right)^{2}+\left(\frac{\partial \Theta}{\partial Y}\right)^{2}\right]+\chi \mathrm{Ha}^{2} \frac{\sigma_{f f}}{\sigma_{f}} V^{2}
\end{aligned}
$$

The quantities with supersubscript " are non-dimensionalized. In the above equation, $\chi$ is the irreversibility factor and is expressed as [22]:

$$
\chi=\frac{\mu_{f} T_{m}}{k_{f}}\left(\frac{V_{0}}{T_{h}-T_{c}}\right)^{2} .
$$

To examine the irreversibility distribution, Bejan num- ber, $\mathrm{Be}$, as a ratio of thermal entropy generation to the total entropy generation is defined as [19]:

$$
\mathrm{Be}=\frac{S_{T}^{\prime}}{S^{\prime}}
$$

The average entropy generation is given as:

$$
S_{\mathrm{avg}}=\int_{0}^{1} \int_{0}^{1} S^{\prime} d X d Y
$$

Then, entropy generation is averaged during the simulation time in the following equation:

$$
\overline{S_{\mathrm{avg}}}=\frac{1}{\tau_{\text {final }}} \int_{0}^{\tau_{\text {final }}} S_{\mathrm{avg}} d t
$$

where $\tau_{\text {final }}$ is the final time of simulation. All of the other kinds of entropy generation in addition to the Bejan and Nusselt numbers are reported in the same method. In order to examine the effect of adding minor volume fraction of ferrite nanoparticles to ferrofluid and applying external magnetic field to the entropy generation and average Nusselt number, the following entropy generation ratio and Nusselt number ratio are defined:

$$
\begin{array}{ll}
{\overline{S_{\mathrm{avg}}}}^{* *}=\frac{\overline{S_{\mathrm{avg}}}(\phi)}{\overline{S_{\mathrm{avg}}}(\phi=0)}, & \overline{\mathrm{Nu}}^{* *}=\frac{\overline{\mathrm{Nu}}(\phi)}{\overline{\mathrm{Nu}}(\phi=0)}, \\
{\overline{S_{\mathrm{avg}}}}^{*}=\frac{\overline{S_{\mathrm{avg}}}(\mathrm{Ha})}{\overline{S_{\mathrm{avg}}}(\mathrm{Ha}=0)}, & \overline{\mathrm{Nu}}^{*}=\frac{\overline{\mathrm{Nu}}(\mathrm{Ha})}{\overline{\mathrm{Nu}}(\mathrm{Ha}=0)} .
\end{array}
$$

\section{Numerical method, grid independency test, and code validation}

The time-dependent incompressible Navier-Stokes and energy equations are numerically solved by an accurate finite volume method based on Chorin-type projection and finite difference approximations for the spatial discretization of a staggered, non-uniform Cartesian grid. The adopted procedure deserves a detailed explanation: A Chorin-type projection method was employed for the decoupling of momentum and continuity equations [23,24]. First, a preliminary velocity field was computed by momentum equation. Then, a Poissontype equation for the pressure was solved, which was obtained by the continuity equation. Finally, preliminary velocity field was projected onto a divergence-free velocity field using the computed pressure. Besides, a second-order Runge-Kutta scheme was utilized for handling of the time derivatives and QUICK upwind scheme was used for discretization of the non-linear terms [25]. The discretized equations were solved by a sparse Cholesky decomposition approach [26]. The 
convergence of numerical results was also implemented and the following criterion was satisfied in each time step:

$$
\sum_{i, j}\left|\Pi_{i, j}^{n}-\Pi_{i, j}^{n-1}\right| \leq 10^{-6}
$$

where the generic variable $\Pi$ represents the set of $U, V$, or $\Theta$, and $n$ indicates the iteration number in one time step. The subscript sequence $(i, j)$ represents the space coordinates of the grid node. The grid independency test study was also carried out to find out the proper grid size. For this, several simulations were performed for three different uniform grids, namely, $100 \times 100$, $128 \times 128$, and $140 \times 140$, and for a typical case of dealing with $\mathrm{Re}=100, \phi=0.1, \mathrm{Ha}=10$, and $\mathrm{Ri}=10$. Minor differences of less than $0.3 \%$ were detected between the produced results by different used grids. Therefore, considering simulated accuracy, the uniform grid of $128 \times 128$ was found enough well to ensure the gridindependent solution. Note that computations were carried out for a time step close to $10^{-3}$, similar to Moumni et al. study [16]. Meanwhile, simulations were continued during 10000 time steps to obtain reliable results.

The presented numerical method had to be validated against published results in the literature to establish the code credibility. For this purpose, two different tests were conducted. The case of natural convection flow in a square enclosure filled with pure fluid was tested first. The results for various pertinent variables were compared with the former results of different approaches in Table 2. It should be noted
Table 2. Comparison of the results obtained by the present numerical method and some others published in the literature.

\begin{tabular}{lcccc}
\hline $\begin{array}{c}\text { Results } \\
\text { obtained by }\end{array}$ & $\begin{array}{c}\text { Rayleigh } \\
\text { number }\end{array}$ & $\mathbf{1 0}^{\mathbf{3}}$ & $\mathbf{1 0}^{\mathbf{5}}$ & $\mathbf{1 0}^{\mathbf{7}}$ \\
\hline Present study & & 3.645 & 34.719 & 148.511 \\
Dixit \& Babu [29] & $U_{\max }$ & 3.652 & 35.521 & 164.236 \\
Kuznik et al. [30] & & 3.636 & 34.962 & 148.768 \\
Moumni et al. [16] & & 3.650 & 34.764 & 148.440 \\
\hline Present study & & 3.695 & 68.590 & 701.658 \\
Dixit \& Babu [29] & $V_{\max }$ & 3.682 & 68.655 & 701.922 \\
Kuznik et al. [30] & & 3.686 & 68.578 & 702.29 \\
Moumni et al. [16] & & 3.698 & 68.603 & 699.571 \\
\hline Present study & & 1.119 & 4.532 & 16.814 \\
Dixit \& Babu [29] & $\overline{N u}$ & 1.121 & 4.546 & 16.790 \\
Kuznik et al. [30] & & 1.117 & 4.518 & 16.408 \\
Moumni et al. [16] & & 1.117 & 4.521 & 16.526 \\
\hline
\end{tabular}

that all of these results were reported in the absence of an external magnetic field. The obtained results of the available code show an acceptable agreement with the others. Furthermore, the code was validated against results of Rahman et al. [27], and Al-Salem et al. [28], while it was a little modified to get similar conditions. Streamlines and isotherms of pure forced convection in the presence of an external magnetic field and inside an enclosed cavity obtained by the present method are compared against the former results in Figure 2. As it can be observed, an excellent agreement with findings of the previous studies is achieved.
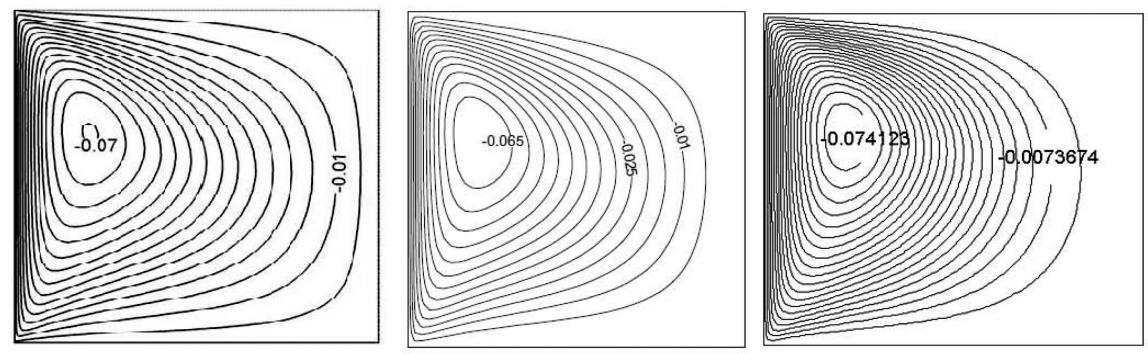

(a)
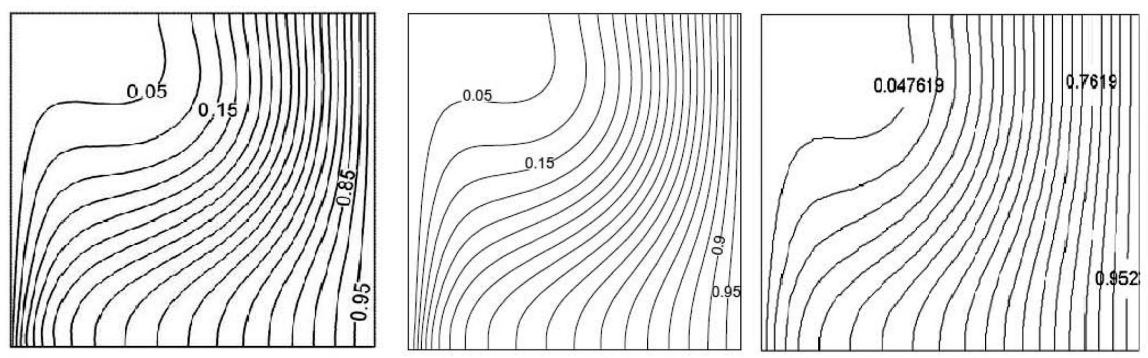

(b)

Figure 2. (a) Streamlines and (b) isotherms of cases with pure forced convection $(\mathrm{Ri}=0)$ and Ha $=10$. The results in the left, middle, and right columns have been obtained by Rahman et al. [27], Al-Salem et al. [28], and the present study, respectively. 


\section{Results and discussions}

MHD mixed convection of ferrofluid in the presence of an external magnetic field and inside a linearly heated two-sided cavity is numerically examined. The computational results are obtained for Richardson number, $\mathrm{Ri}$, ranging from 0.01 to 10 , Hartmann number, Ha, ranging from 10 to 100 , and solid volume fraction, $\phi$, ranging from 0 to 0.2 . Besides, two kinds of external magnetic field are applied: constant and time periodic, with different periods, $\tau_{B}=\frac{t_{B} V_{0}}{L}$, ranging from 1 to 10 , and different phase deviations, $\varphi$, ranging from 0 to $\frac{\pi}{3}$. It should be noted that all of the results are reported at stationary-state conditions.

\subsection{Effect of Richardson number Ri}

Figures 3 and 4 represent the effect of Richardson number on streamline and isotherm contours for cavity filled with ferrofluid, when $\mathrm{Ha}=25, \phi=0.1$, and $\operatorname{Re}=100$. In particular, Figure 3(a) and (b) display the influence of external magnetic field period on fluid flow and isotherm patterns. The flow is characterized by two counter-rotating cells localized in the proximity of moving walls. In fact, the fluid rises up from the linearly heated bottom wall due to buoyant forces and flows down along the cold side. The competition between forced flow provoked by moving walls and buoyancy driven flow formed counter-rotating cells. The variation of thermal buoyancy force along the linearly heated wall caused the rotating cells to have different sizes. Additionally, the external magnetic
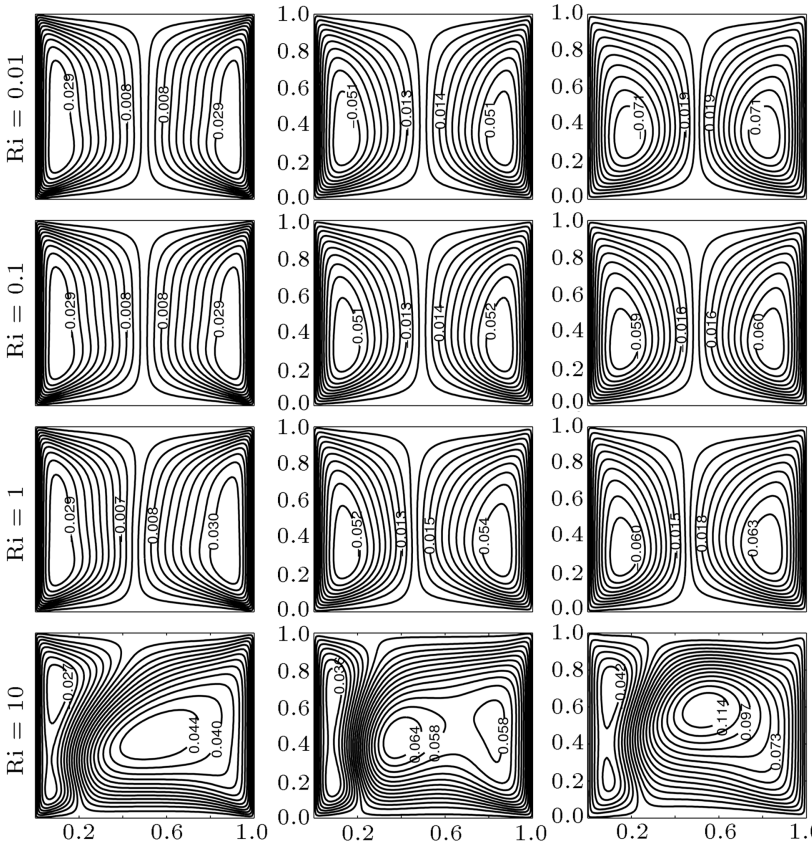

(a)

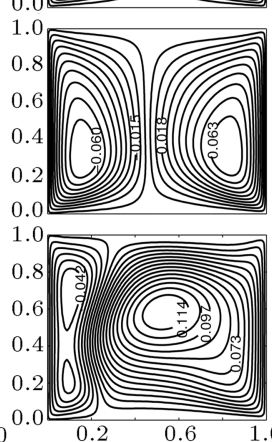

field had the tendency to slow down the movement of the buoyancy induced flow in the cavity, which will be discussed in the next section. Figure 3(a) shows that when the flow is dominated by forced convection $\mathrm{Ri} \ll 1$, fluid flow is characterized by the moving of the side walls and a clear symmetry is observed between counter-rotating cells. Nevertheless, by applying a time-periodic magnetic field, the size of cells core shrinks and the magnitude of cores vortices, i.e. stream functions, increases. According to the role of magnetic field in reducing the fluid intensity, it can be claimed that the fluid experiences a smaller Lorenz force when it is affected by a time-periodic magnetic field. When natural and forced convections become equal, $\mathrm{Ri}=1$, the role of linearly heated wall is intensified and the symmetry of the fluid flow starts to change. These variations are more pronounced when the effect of natural convection dominates forced convection effect, $\mathrm{Ri}>1$. In respect to the linear heating of the horizontal wall, the right cell becomes larger and it has a tendency to compact the left cell. However, the role of time-periodic magnetic fields in increasing the absolute values of the stream function is still observed.

Among the cases under periodic magnetic fields, the case with the longer period has usually registered higher vortex values. This fact demonstrates that the cases under a time-periodic magnetic field with longer periods experience less effective magnetic field or smaller Lorenz force in comparison to the others. Figure 3(b) displays the effects of Richardson number
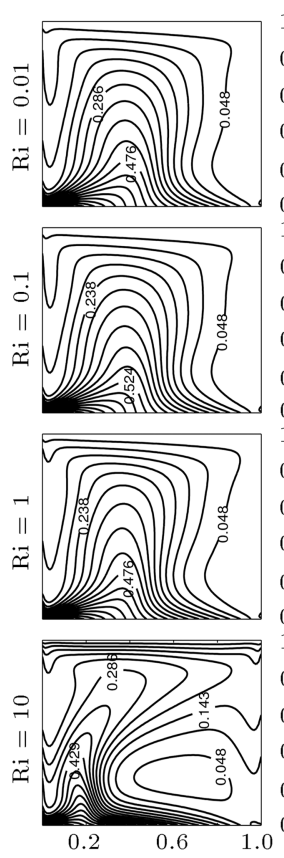

Figure 3. Top and bottom figures, respectively, present (a) streamlines and (b) isotherm contours for ferrofluid filled cavity at different Richardson numbers. The left column presents cases under constant magnetic field $(\mathrm{Ha}=25)$, while middle and right columns present cases under periodic magnetic fields with $\tau_{B}=5$ and $\tau_{B}=10$, respectively. 

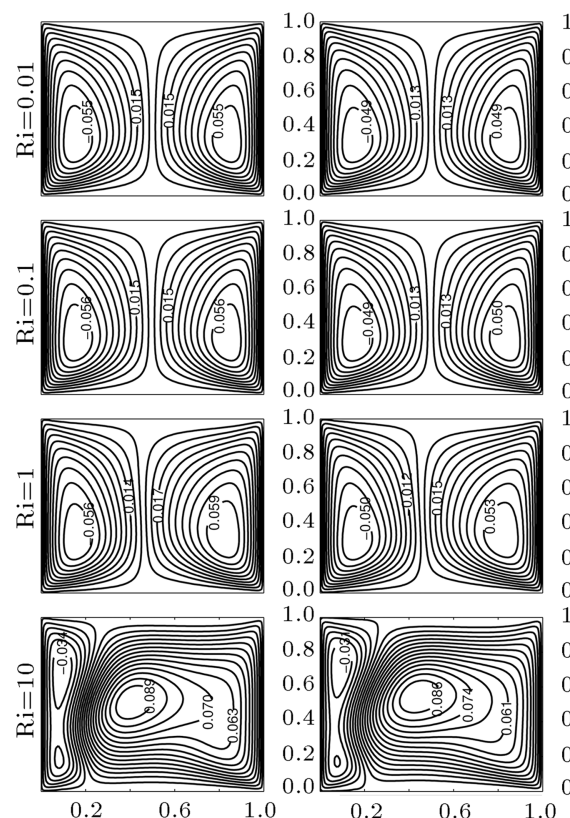

(a)
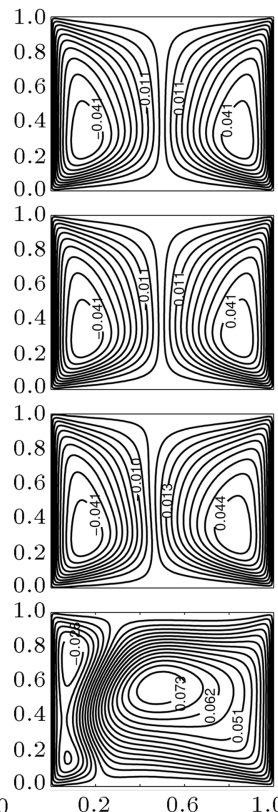
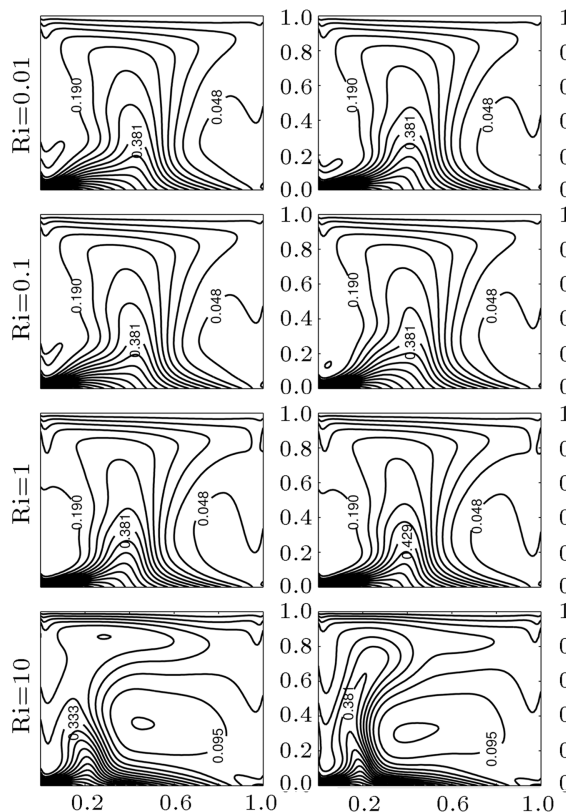

(b)

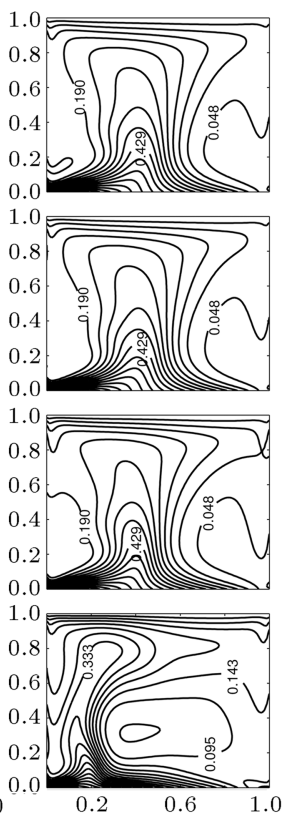

Figure 4. Top and bottom figures, respectively, present (a) streamlines and (b) isotherm contours for ferrofluid filled cavity at different Richardson numbers. The left, middle, and right columns present cases under time-periodic magnetic field with $\varphi=\frac{\pi}{6}$, $\frac{\pi}{4}$, and $\frac{\pi}{3}$, respectively, when $\operatorname{Re}=100, \mathrm{Gr}=10^{5}$, and $\phi=0.1$ in all cases.

and period of time-periodic magnetic field on isotherm contours. The isotherm maps are characterized by a thermal plume-like phenomenon, which was accentuated with increasing Richardson number. It can also be seen that the temperature gradient at the vicinity of linearly heated wall increases by increasing the Ri value. This is well explained by the stretched and tightened thermal boundary layers as $\mathrm{Ri}$ increases, observed before by Moumni et al. [16] for $\mathrm{Cu}$-water nanofluid, which is due to increase in natural convection importance against forced convection.

The effect of phase deviation on fluid flow and temperature distribution of forced, mixed, and natural convection of ferrofluid can be studied in Figure 4, where streamlines and isotherms for cases under the periodic magnetic fields with certain period $\left(\tau_{B}=5\right)$, constant amplitude $(\mathrm{Ha}=25)$, and different phase deviations, i.e. $\frac{\pi}{6}, \frac{\pi}{4}$, and $\frac{\pi}{3}$, are illustrated. Figure 4(a) shows that the influence of phase deviation on fluid flow seems to be insignificant. Moreover, Figure 4(b) shows that phase deviation has no significant effect on isotherm maps. Nevertheless, it can be seen that the cases under periodic magnetic fields with larger phase deviations have registered smaller stream functions. It was mentioned earlier that increasing external magnetic field reduces the intensity of MHD mixed convection fluid flow. Therefore, the cases under periodic magnetic fields with larger phase angels should strengthen Lorenz force. This can be attributed to the temporal variations of magnetic field during the simulation time, which was denoted in Figure 5(a) by solid arrows to help better understanding. The external magnetic fields with smaller phase angles provide smaller Lorenz forces at initial and final time steps. Hence, the cases under time periodic magnetic fields with smaller phase deviations have larger vortex values and stream functions at the final time.

The influence of Richardson number and period of the alternating magnetic field on the average Nusselt number is depicted in Figure 5(b). It can be seen that $\overline{\mathrm{Nu}}$ is increased monotonically with an increase of Richardson number. This fact was also observed before by Nayak et al. [18] and attributed to increase of buoyancy effect with increasing $\mathrm{Ri}$, which enhances the thermal boundary layer thickness and, thus, improves heat transfer. Applying time-periodic magnetic field, which usually has smaller momentary values than constant magnetic field, also improves heat transfer across the cavity. Meanwhile, the cases under a periodic magnetic field with longer period have larger $\overline{\mathrm{Nu}}$ values.

Temporal variation of the applied external magnetic field has also influenced entropy generation is achieved. Figure 5(c) shows that the average entropy generation is decreased with the increasing of Richardson number. It was seen earlier that the thermal boundary thickness as well as the fluid intensity were augmented with further enhancement of Richardson number. This means that the increasing of the Richardson number increases thermal buoyancy forces, improves convection, and therefore reduces conductive mode of heat transfer within the cavity. One of the important terms of entropy generation is the term of 


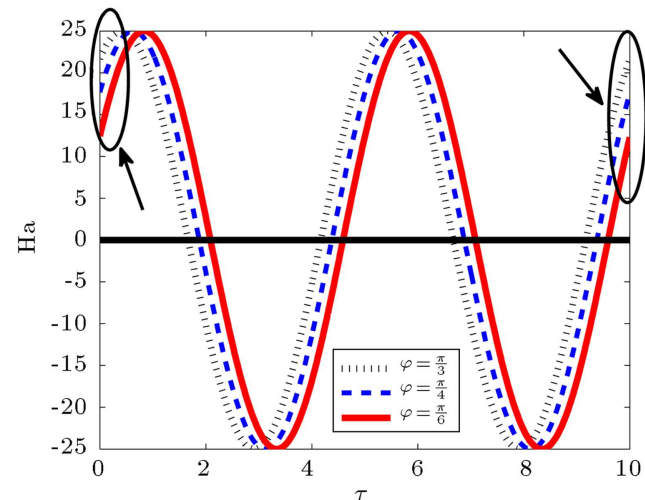

(a)

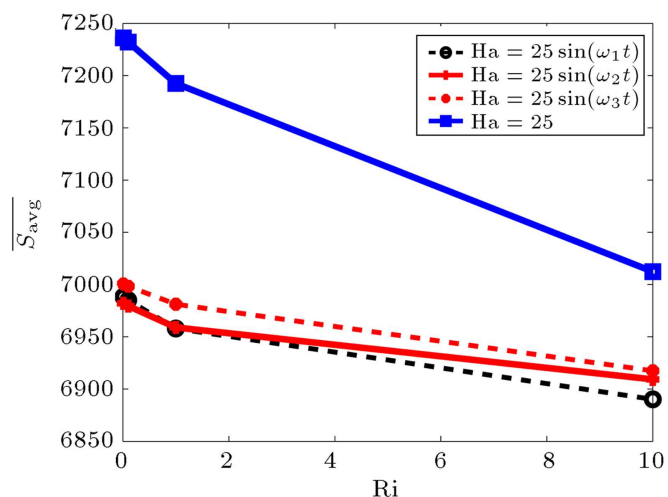

(c)

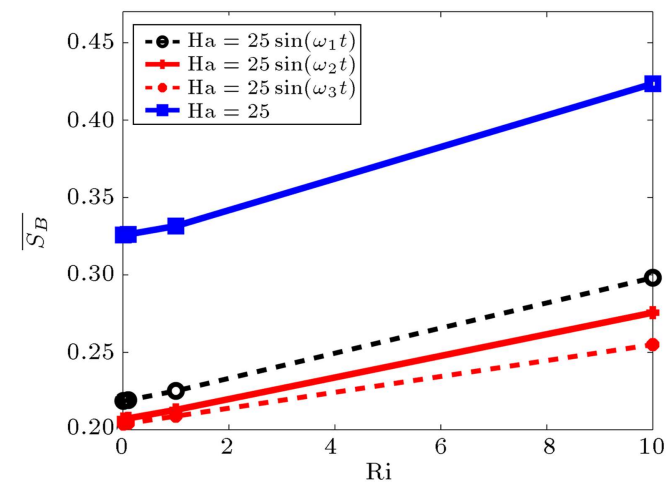

(e)

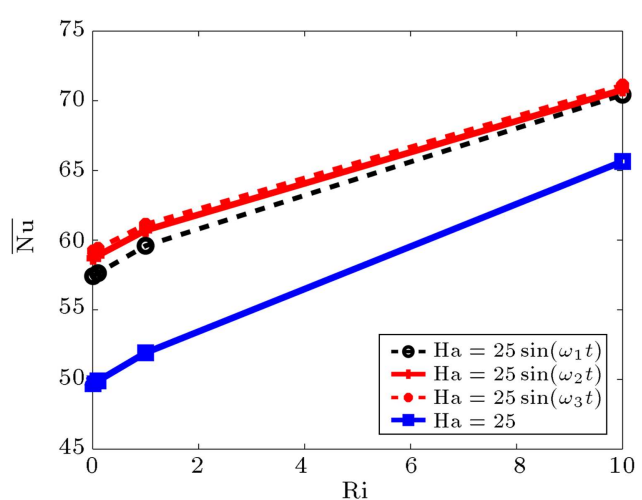

(b)

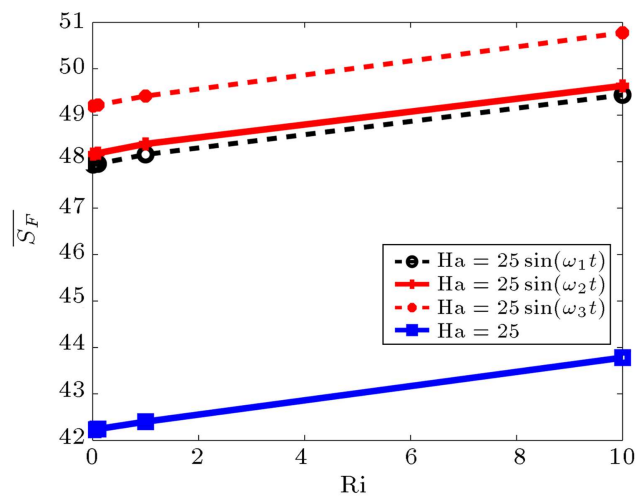

(d)

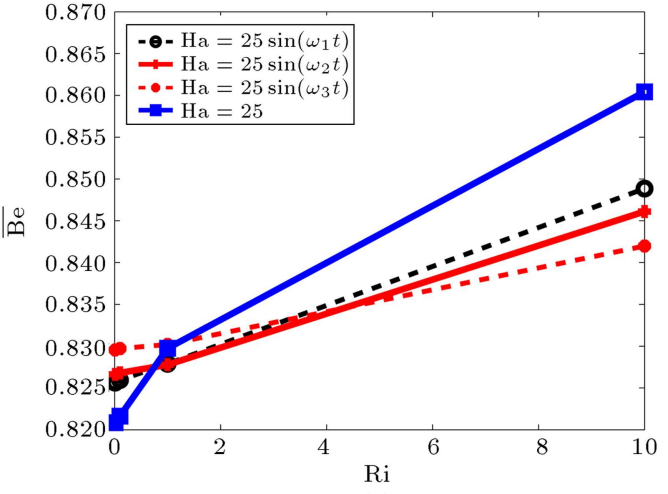

(f)

Figure 5. (a) Temporal variations of time-periodic Hartmann numbers with various phase deviations, when amplitude of variations is kept constant at 25. Variations of average (b) Nusselt number, (c) entropy generation, (d) viscous dissipation entropy generation, (e) magnetic entropy generation, and (f) Bejan number as a function of Richardson number for cavity filled with ferrofluid under constant magnetic field $(\mathrm{Ha}=25)$ and time-periodic magnetic fields with $\tau_{B_{1}}=1, \tau_{B_{2}}=5$, and $\tau_{B_{3}}=10$, where $\phi=0.1$.

thermal irreversibility, which was directly related to the conductive mode of heat transfer, see Eqs. (20)-(24). Therefore, although the enhancement of Richardson number increases the intensity of buoyancy driven flow, it causes a sensible reduction in thermal irreversibility. This figure also shows that applying time-periodic magnetic field makes a reduction in the entropy generation. Meanwhile, although the period of magnetic field has not significantly influenced the entropy generation reduction, cases with shorter periods have registered smaller $\overline{S_{\text {avg }}}$ values. Accordingly, it can be claimed that applying time-periodic magnetic field with shorter period can be one of the Entropy Generation Minimization (EGM) techniques in MHD convection of ferrofluid flows.

The effect of Richardson number on the enhancement of fluid intensity is well observed in Figure 5(d), where the fluid friction entropy generation is illustrated as a function of Richardson number. The cases under periodic magnetic fields usually have larger $\overline{S_{F}}$ values. The influence of Richardson number and periodic variation of applied magnetic field on the magnetic entropy 


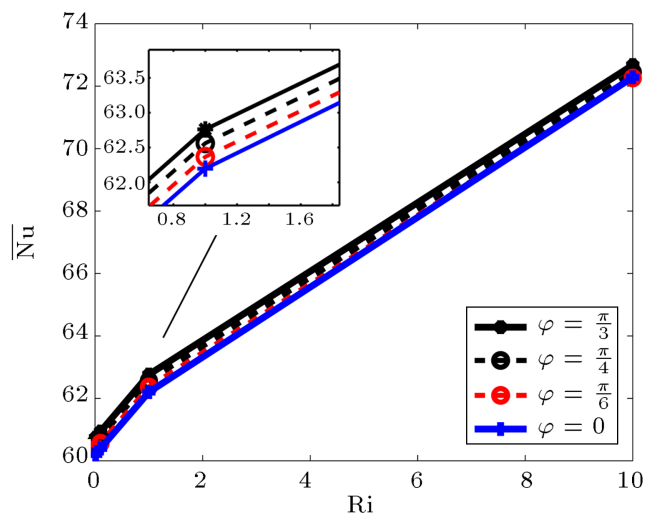

(a)

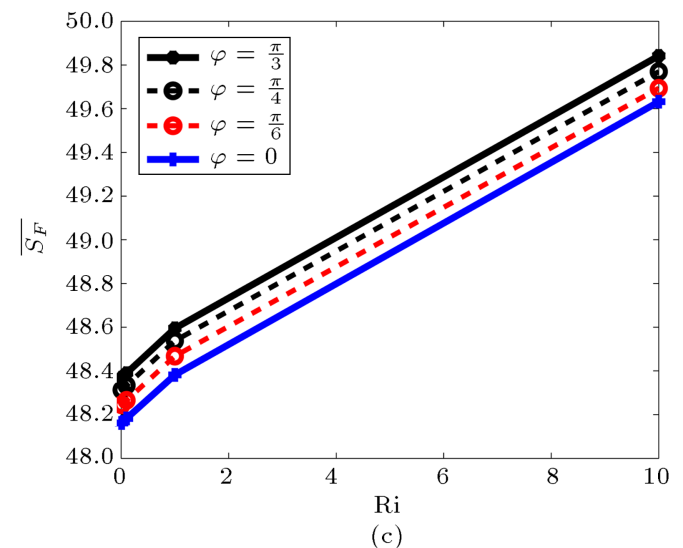

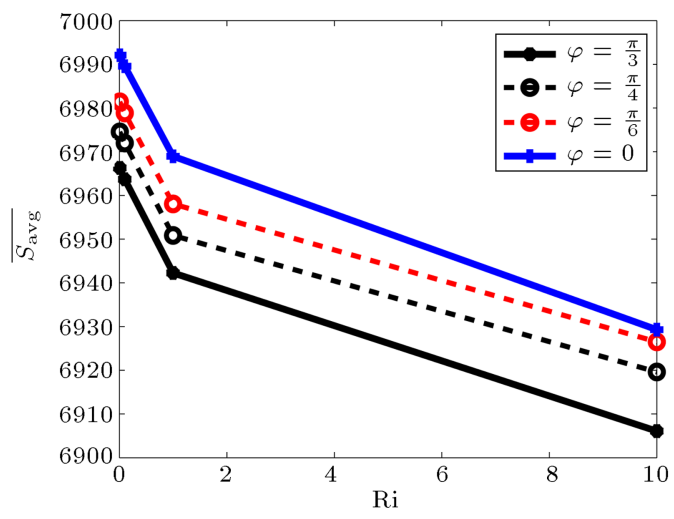

(b)

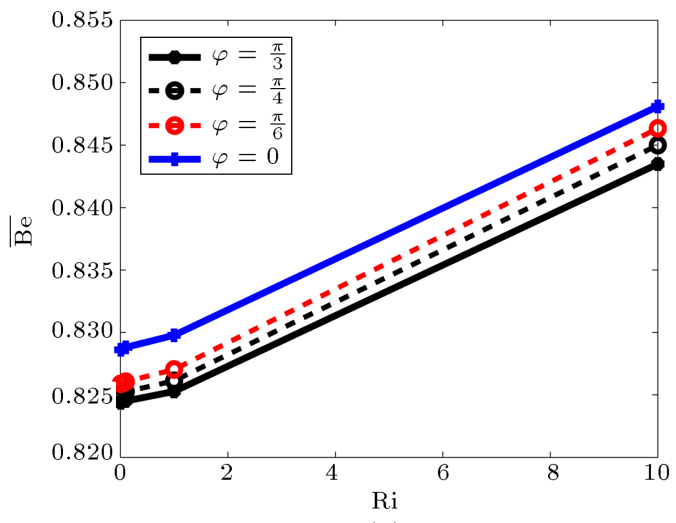

(d)

Figure 6. Variations of average (a) Nusselt number, (b) entropy generation, (c) viscous dissipation entropy generation, and (d) Bejan number as a function of Richardson number for cavity filled with ferrofluid under time-periodic magnetic fields with various phase deviations, where $\phi=0.1$. Amplitude of time-periodic magnetic field is kept constant at 25 .

generation is depicted in Figure 5(e). It can be seen that increase in fluid intensity caused by Richardson number augmentation makes an enhancement in $\overline{S_{B}}$ values. Moreover, the cases under periodic magnetic field usually have lower $\overline{S_{B}}$ values due to experiencing lower effective magnetic field. The variation of average Bejan number, $\overline{\mathrm{Be}}$, at different values of Richardson number is presented in Figure $5(\mathrm{f})$. First, it can be seen that the values of $\overline{\mathrm{Be}}$ are higher than 0.8 , which represents that the thermal irreversibility dominates the other irreversibilities. $\overline{\mathrm{Be}}$ increases by increasing Richardson number and temporal variations of external magnetic field have sufficiently influenced the $\overline{\mathrm{Be}}$ variation, especially at larger $\mathrm{Ri}$ values. At smaller Richardson numbers, $\mathrm{Ri}<1$, the cases under timeperiodic magnetic field have larger $\overline{\mathrm{Be}}$ values, while the opposite is observed in cases with $\mathrm{Ri} \geq 1$. The influence of external magnetic field on both convective heat transfer reduction and conductive heat transfer enhancement was intensified by increasing the role of natural convection and increasing the value of Richardson number. Therefore, the contribution of thermal irreversibility and conductive mode of heat transfer in the total entropy generation in cases with time-periodic magnetic field was decreased with increasing value of
Richardson number. It should be again noted the cases under periodic magnetic fields experience smaller effective Lorenz force than the cases under constant magnetic fields.

The influence of phase deviation on the average Nusselt number is presented in Figure 6(a). As can be seen, the increase in phase deviation caused a slight enhancement in the convective heat transfer. On the other hand, Figure 6(c) and (d) shows that the cases under periodic magnetic field with larger phase deviations have smaller and larger $\overline{S_{\text {avg }}}$ and $\overline{S_{F}}$ values, respectively. This fact is attributed again to the effective Lorenz force applied by external magnetic field. The variation of average Bejan number as a function of Richardson number for different cases with various phase deviations is depicted in Figure 6(d). It is evident that Bejan number is still more than 0.8 and thermal irreversibility is dominant with respect to the other fluid irreversibilities. In addition, cases with larger phase deviations have smaller average Bejan numbers.

\subsection{Effect of Hartmann number, Ha}

The influence of Hartmann number and the period of external magnetic field on flow patterns and isotherm 

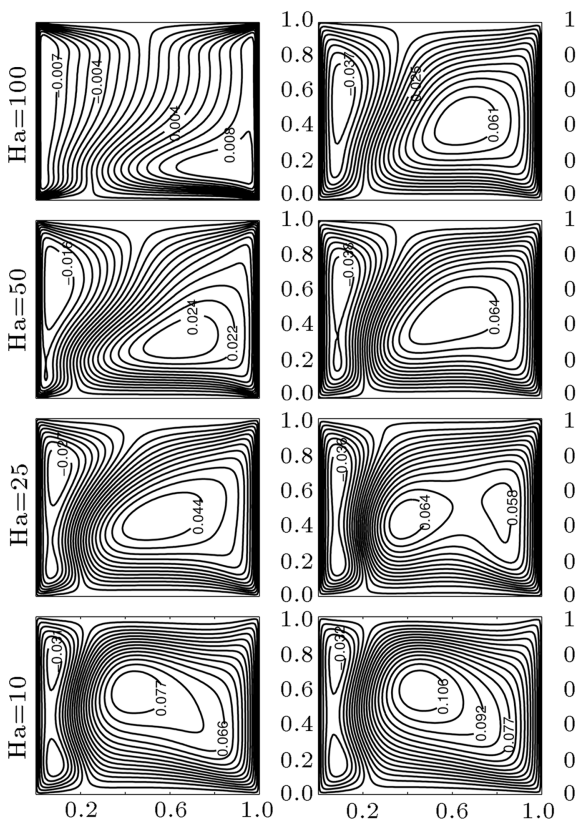

(a)
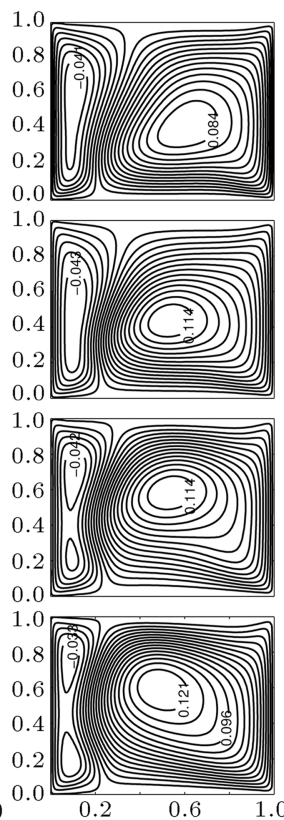
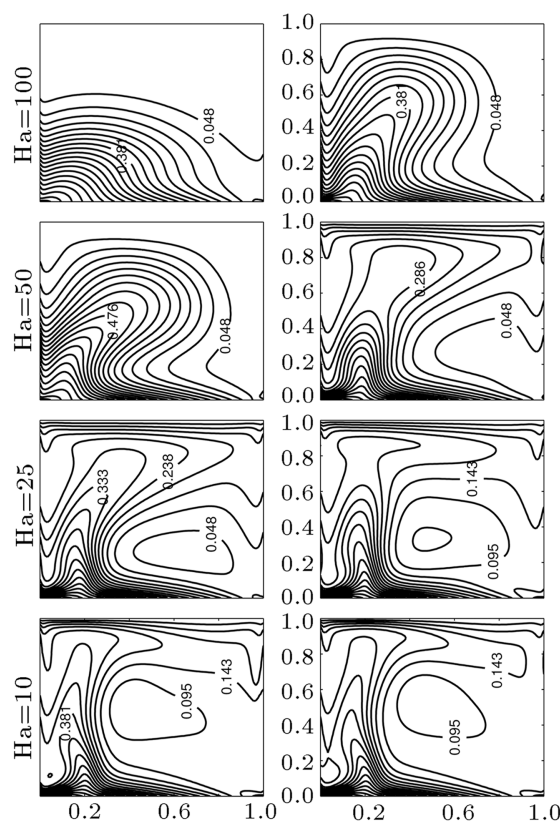

(b)

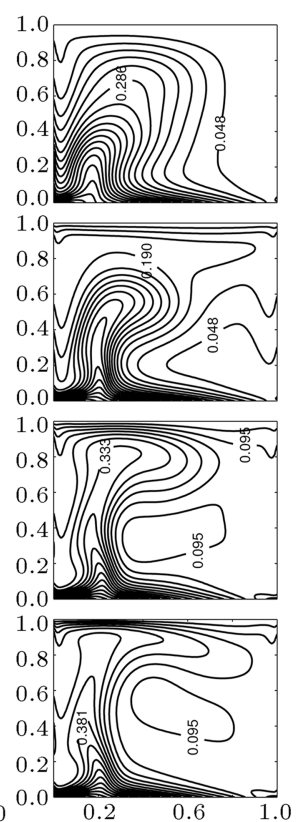

Figure 7. Top and bottom figures, respectively, present (a) streamlines and (b) isotherm contours for ferrofluid filled cavity at different Hartmann numbers. The left column presents cases under constant magnetic field, while the middle and right columns present cases under periodic magnetic fields with $\tau_{B}=5$ and $\tau_{B}=10$, respectively, when $\mathrm{Ri}=10$, and $\phi=0.1$ in all cases.

contours is shown in Figure 7, where $\mathrm{Ri}=10$ and $\phi=$ 0.1 . It should be noted that natural convection is the dominant convection mechanism here due to the utilized value of Richardson number. It was noticed earlier that the external magnetic field suppresses the flow field and affects the counter-rotating cells. This fact well remarks here, whereby the stream function values are reduced by further enhancement of Hartmann number. In fact, Lorenz force has a resistance effect against thermal buoyancy forces and, thus, has a tendency to slow down the buoyancy driven flow. The influence of Hartmann number and periodic magnetic field on isotherm contours is studied in Figure 7(b). This figure displays that the corresponding thermal layer near the heated wall becomes less concentrated and the isotherms become parallel to the bottom horizontal wall with the increase of Hartmann number. In other words, increasing Ha values causes the convection heat transfer mode to become dominated by the conduction mode, and cavity becomes a quasi-conduction domain. The effect of Lorenz force on flow field and temperature distribution was also observed before by Pekmen \& Tezer-Sezgin [31], El-Shehabey \& Ahmed [32], and Mehrez et al. [22] in other different thermal systems containing nanofluids. However, if streamlines and isotherm contours are juxtaposed to each other, it can be seen that the size of rotating cells is directly related to the competition of thermal buoyancy forces and mechanical forces induced by moving walls. In the case under largest Hartmann number, the right cell has both the smallest size and the smallest stream function with respect to the other cases. The separation of two rotating cells at the bottom half of the cavity is caused by linear heating of the bottom wall. The buoyancy forces at the right of the bottom half of the cavity are weaker than the cross side and, thus, the right and left cells usually have larger and smaller sizes, respectively, when the flow is dominated by natural convection. The natural convection within the cavity is accentuated with increasing Hartmann number, whereby the sizes of right and left cells are reduced and increased with the augmentation of Hartmann number, respectively. It is evident that applying time-periodic magnetic fields increases the stream function values and the cases under fields with longer periods have larger stream functions. Moreover, applying periodic field sufficiently affects the thermal boundary layers, especially in cases under $\mathrm{Ha}=100$, whereby parallel thermal layers are distorted by utilizing the alternating magnetic fields.

The effect of Hartmann number on the average Nusselt number at the bottom heated wall is shown in Figure 8(a). From this figure, it is observed that $\overline{\mathrm{Nu}}$ goes down almost rapidly with increasing Ha values. As the intensity of magnetic field increases, conductive heat transfer is enhanced and, thus, convection mechanism into the cavity vanishes. The cases under periodic external fields with respect to the cases with constant Hartmann number experience smaller Lorenz forces and, thus, have larger $\overline{\mathrm{Nu}}$ values. Meanwhile, the cases under periodic magnetic field with longer 


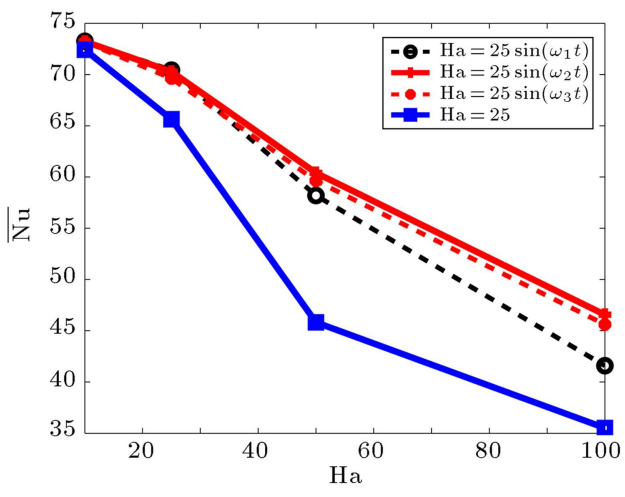

(a)

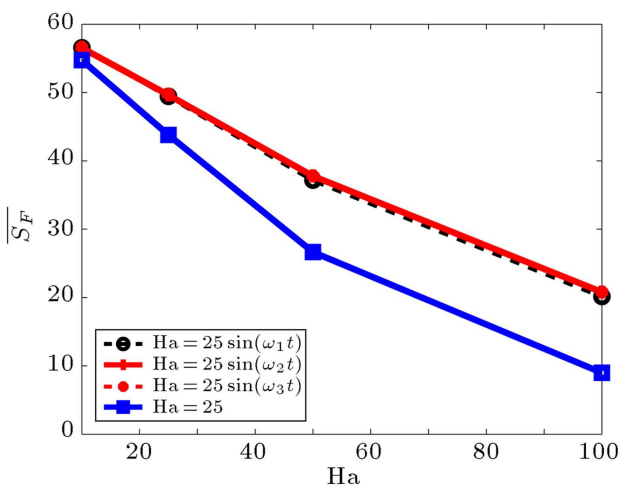

(c)

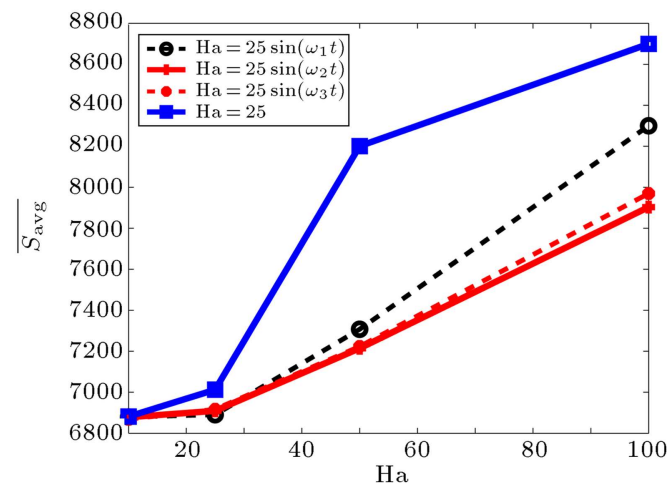

(b)

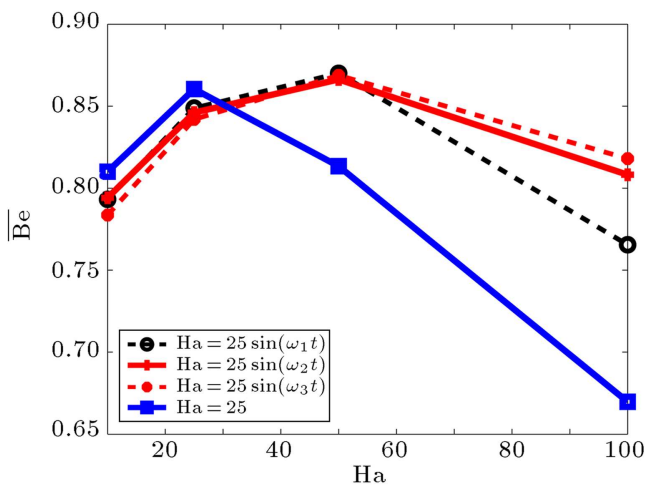

(d)

Figure 8. Variations of average (a) Nusselt number, (b) entropy generation, (c) entropy generation due to fluid friction, and (d) Bejan number as a function of Hartmann number for cases under constant magnetic field and time-periodic magnetic field with various periods, where $\omega=\frac{2 \pi}{\tau_{B}}, \tau_{B_{1}}=1, \tau_{B_{2}}=5, \tau_{B_{3}}=10, \mathrm{Ri}=10$, and $\phi=0.1$.

periods have larger Nusselt numbers. The influence of Hartmann number and periodic magnetic field on the entropy generations and different fluid irreversibilities is illustrated in Figure 8(b)-(d). It has been mentioned that the conductive heat transfer mode is improved with increasing Hartmann number, while Lorenz force usually suppresses fluid intensity. Figure 8(b) and (c) demonstrate that although fluid friction irreversibility, $\overline{S_{F}}$, is reduced monotonically by Hartmann number, Figure 8(c), increasing irreversibility due to thermal effect causes an enhancement in the average entropy generation. According to their properties, smaller effective Lorenz force, the cases under time-periodic magnetic field have smaller and larger $\overline{S_{\text {avg }}}$ and $\overline{S_{F}}$ values, respectively. The variation of average Bejan number as a function of Hartmann number is illustrated in Figure 8(d). It can be seen that $\overline{\mathrm{Be}}$ for cases under constant Hartmann number is increased with increasing Hartmann number up to the value of 25 and then it is decreased monotonically with further increasing of Ha. The turning point for the cases under time-periodic magnetic fields changes to $\mathrm{Ha}=$ 50. Moreover, those cases have larger $\overline{\mathrm{Be}}$ values with respect to the cases with constant Hartmann number.

The influence of phase deviation on average Nusselt number and various irreversibilities is illus- trated in Figure 9. First, it can be seen that phase deviation has not sufficiently affected heat transfer or fluid irreversibilities. However, the cases under timeperiodic magnetic fields with larger phase deviations have slightly larger and smaller $\overline{\mathrm{Nu}}$ and $\overline{S_{\text {avg }}}$ values, respectively. In contrast, Figure $9(\mathrm{~d})$ shows that the cases with the smallest phase deviations have larger $\overline{\mathrm{Be}}$ values. The higher values of magnetic field at the initial and final times, Figure 5(a), are again responsible for this variation.

The variation rates of average Nusselt number and entropy generation in ferrofluid compared to pure fluid are depicted in Figure 10. Figure 10(a) shows that the value of $\overline{\mathrm{Nu}}^{* *}$ is larger than unity for Hartmann numbers smaller than 25. This indicates that the existing $10 \%$ of highly electrically conductive nanoparticles in the pure fluid make an enhancement in convective heat transfer when $\mathrm{Ha} \leq 25$. When $\mathrm{Ha}>25$, the existing nanoparticles in the presence of external magnetic field reduce convective heat transfer of ferrofluid. Meanwhile, the destructive effect of high-volume metallic nanoparticles on convection current is intensified by increasing external magnetic field. Figure 10(a) and (b) show that heat transfer of ferrofluid against pure fluid is reduced slowly in the cases under periodic magnetic fields in comparison with the cases under constant 


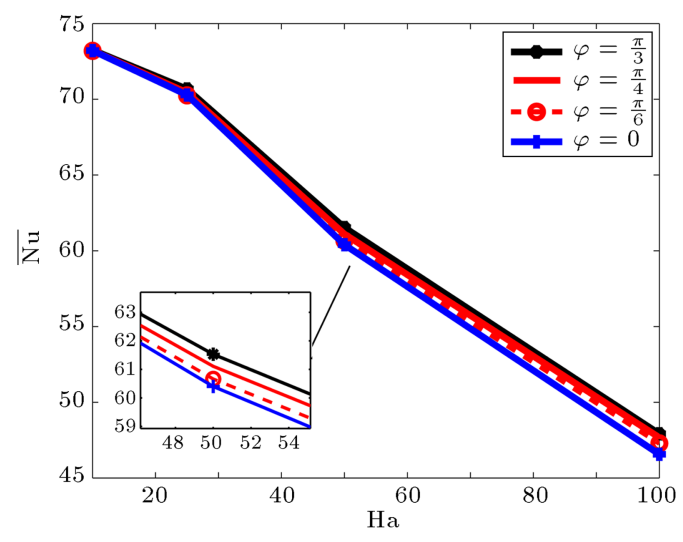

(a)

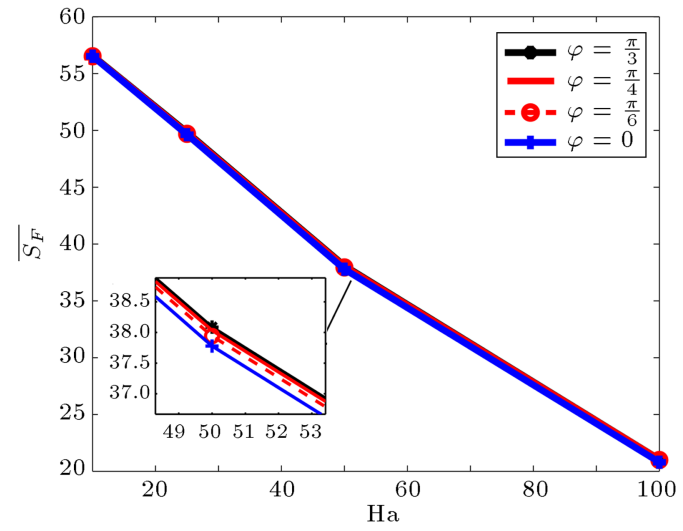

(c)

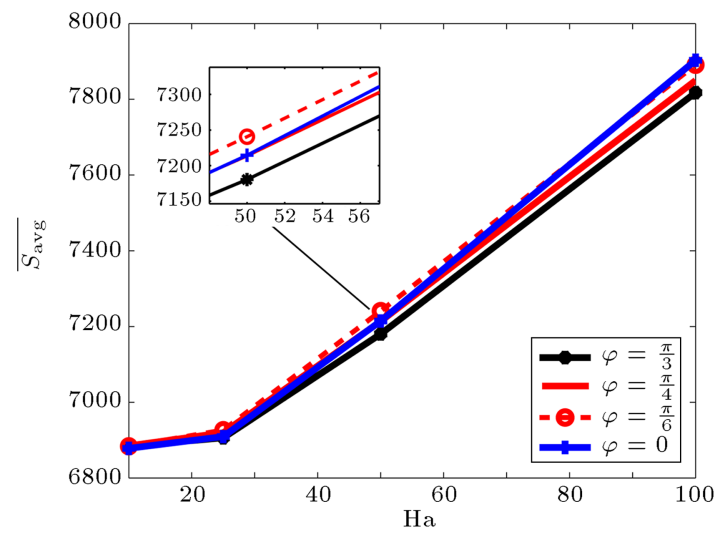

(b)

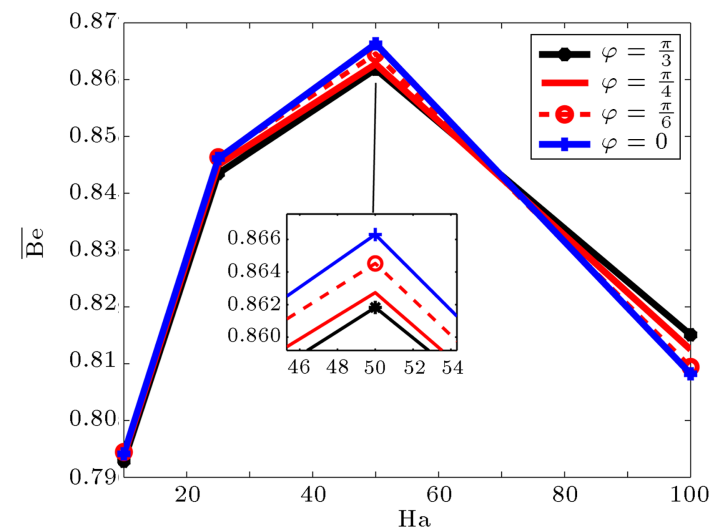

(d)

Figure 9. Variations of average (a) Nusselt number, (b) entropy generation, (c) entropy generation due to fluid friction, and (d) Bejan number as a function of Hartmann number for cases under periodic magnetic field with amplitude of 25 and different phase deviations, when $\mathrm{Ri}=10$ and $\phi=0.1$.

Hartmann number. In addition, longer period causes a smaller reduction rate, while the influence of phase deviation seems to be insignificant. Figure 10(c) and (d) show that those metallic nanoparticles in the presence of an external magnetic field make an enhancement in the total entropy generation. The existence of those nanoparticles in the base fluid sufficiently improves the electrical conductivity of ferrofluid, which results in an entropy generation enhancement. The cases with longer periods or larger phase deviations have the smallest $\overline{S_{\text {avg }}} * *$ values.

\subsection{Effect of nanoscale ferroparticle volume fraction $\phi$}

The influence of solid volume fraction of ferroparitcles on the heat transfer is examined in Figure 11(a), where the variations of the average Nusselt number as a function of the values of $\phi$ for a certain Richardson number $(\mathrm{Ri}=10)$ are depicted. It can be seen that heat transfer within the cavity is improved with increasing value of $\phi$ up to $\phi=0.1$, and then it is reduced with adding more volume fraction of nano-meter sized ferroparticles to the base fluid. The existence of the highly thermally conductive nanoparticles in the base fluid improves the thermal conductivity of the ferrofluid and causes the convective heat transfer to penetrate mush deeper into the cavity. On the other hand, adding more highly electrically conductive metallic nanoparticles to the base fluid improves electrical conductivity of the ferrofluid. The enhancement of the electrical conductivity in the presence of an external magnetic field increases and decreases conductive and convective modes of heat transfer within the cavity, respectively. Most of the previous investigators have studied the existence of less than $10 \%$ nanoparticles in the nanofluid and denoted that increasing solid volume fraction makes an enhancement in MHD natural convection [33,34]. Nevertheless, Nanna experimentally observed that convective heat transfer was reduced with adding more than $10 \%$ metallic nanoparticles to the base fluid [35]. Figure 11(a) also shows that the variations of $\overline{\mathrm{Nu}}$ in cases under periodic magnetic fields seem to be similar and heat transfer within the cavity is improved by decreasing the period of alternating magnetic field. Moreover, applying time-periodic magnetic field makes an enhancement in convection heat transfer within the cavity.

The existing metallic nanoparticles in the base 


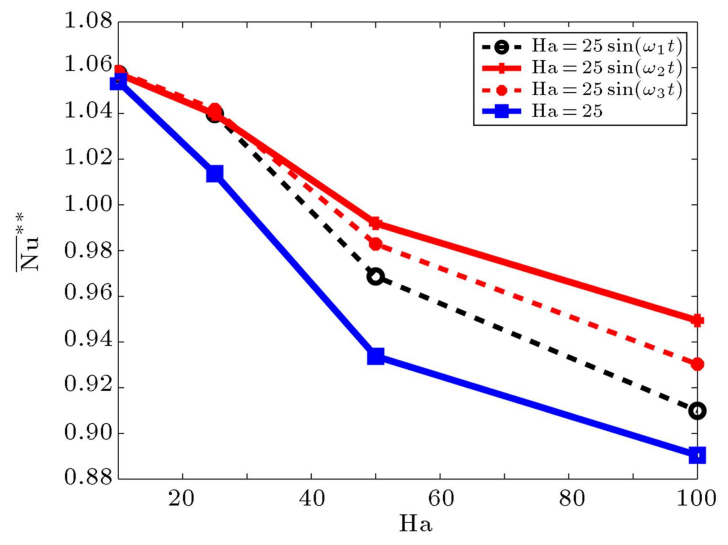

(a)

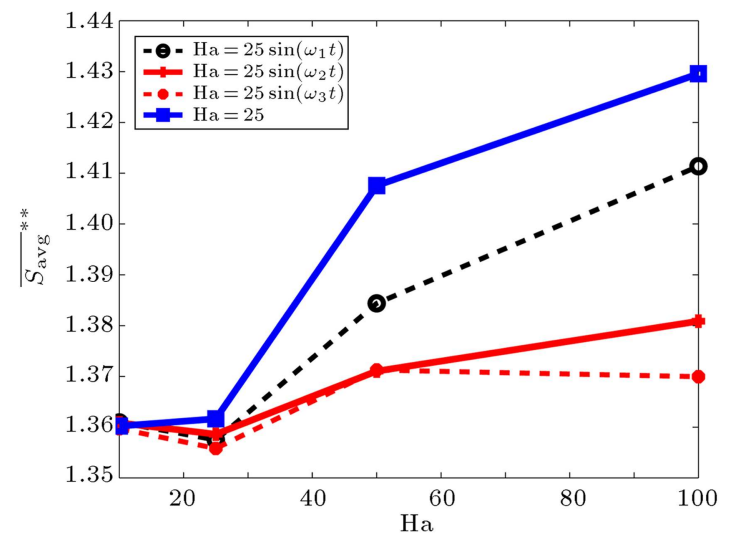

(c)

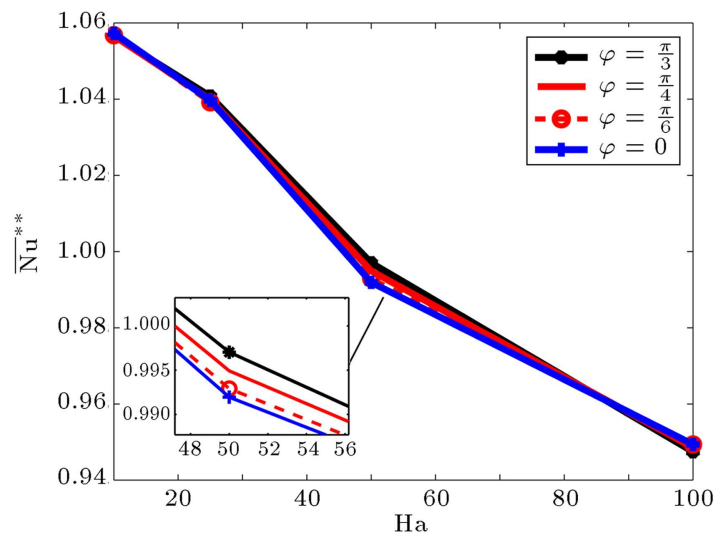

(b)

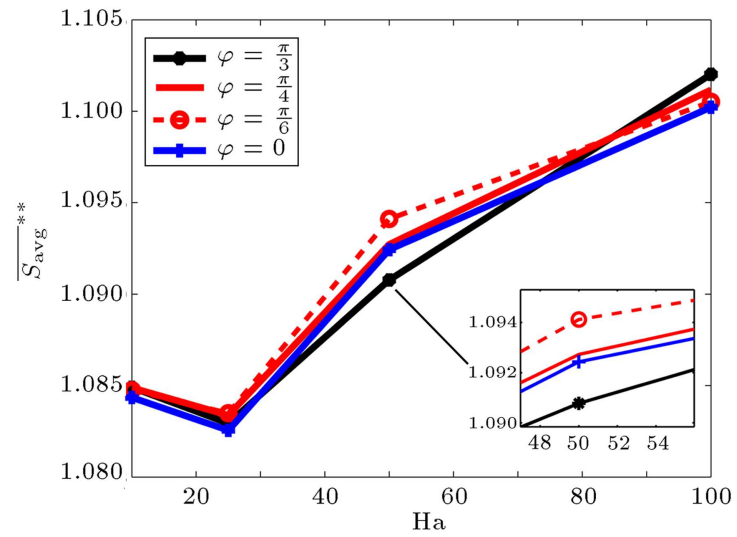

(d)

Figure 10. Variation rates of (a) \& (b) average Nusselt number and (c) \& (d) average entropy genera- tion of ferrofluid with $\phi=0.1$ with respect to pure fluid as a function of Hartmann number, where $\omega=\frac{2 \pi}{\tau_{B}}, \tau_{B_{1}}=1, \tau_{B_{2}}=5, \tau_{B_{3}}=10$, and $\mathrm{Ri}=10$.

fluid also influence different irreversibilities of the ferrofluid. Figure 11(b) shows that the total entropy generation is increased by adding more volume fraction of nanoparticles to the base fluid. The existence of those highly electrically conductive nanoparticles in the base fluid improves both conductive mode of heat transfer and thermal irreversibility of the fluid. Besides, increasing the volume fraction increases viscous dissipation and, thus, improves irreversibility due to fluid friction. Therefore, as can be seen in Figures 11(b) $\& 11(\mathrm{c})$, both $\overline{S_{\mathrm{avg}}}$ and $\overline{S_{F}}$ are increased with further increase in the value of $\phi$. On the other hand, it is observed that external magnetic field suppresses fluid intensity; thus, similar to the previous section, cases under periodic magnetic field have larger values of $\overline{S_{F}}$ due to experiencing smaller Lorenz force. The variation of average Bejan number as a function of solid volume fraction is depicted in Figure 11(d). First, it can be seen that $\overline{\mathrm{Be}}$ is still larger than 0.8 , which implies the dominant effect of thermal irreversibility with respect to the other irreversibilities. Besides, $\overline{\mathrm{Be}}$ increases by further increase in solid volume fraction of nanoparticles. This is attributed again to the increase in conduction heat transfer mode because of existing metallic nanoparticles in the presence of external magnetic field. The cases under time-periodic magnetic fields have smaller $\overline{\mathrm{Be}}$ values due to the same reason. Among them, the cases with longer periods have smaller average Bejan numbers.

The effect of phase deviation on the convection heat transfer and fluid irreversibilities when solid volume fraction varied is illustrated in Figure 12. Figure 12(a) shows that cases with larger phase deviations have larger average Nusselt numbers. This was also observed and discussed in the previous sections. Figure 12(b)-(d) indicate that phase deviation has not a significant effect on fluid irreversibilities or Bejan number. Nevertheless, the cases with smaller phase deviations have slightly larger $\overline{\mathrm{Be}}$ values. The influence of period and phase deviation on $\overline{\mathrm{Nu}}^{*}$ and ${\overline{S_{\mathrm{avg}}}}^{*}$ values is illustrated in Figure 13. First, it can be seen that all of the $\overline{\mathrm{Nu}}^{*}$ and $\bar{S}_{\mathrm{avg}} *$ values are smaller and larger than unity, respectively. In other words, applying an external magnetic field decreases and increases the heat transfer and the entropy generation, respectively. Nevertheless, the role of magnetic field in both heat transfer reduction and entropy generation enhancement is augmented with further increase in solid volume fraction 


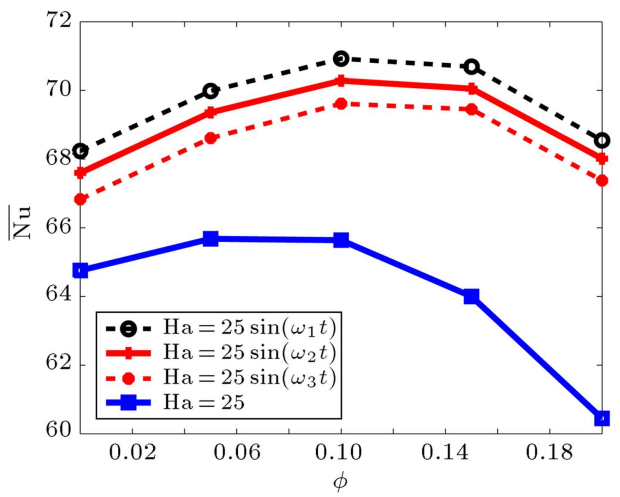

(a)

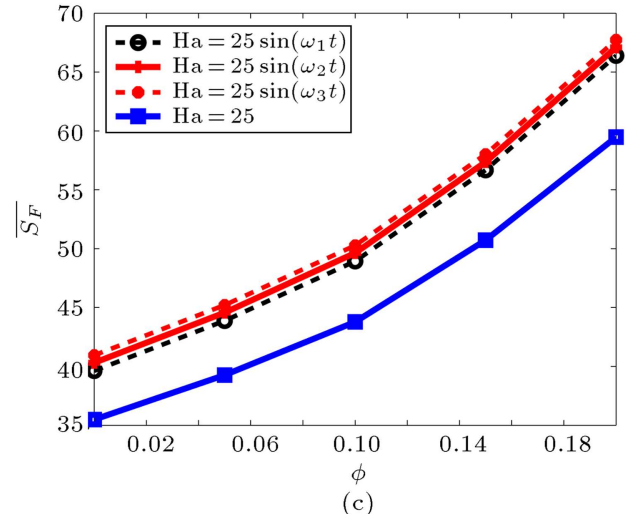

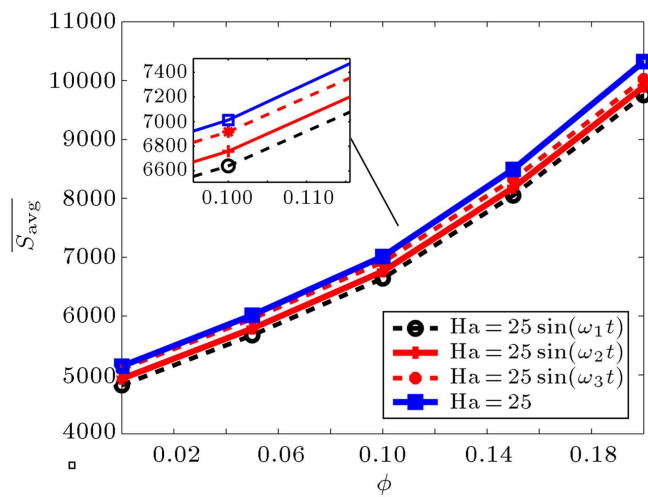

(b)

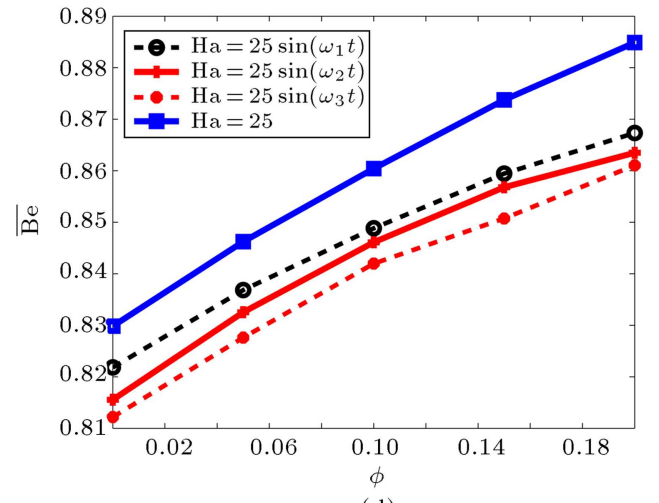

(d)

Figure 11. Variations of average (a) Nusselt number, (b) entropy generation, (c) entropy generation due to fluid friction, and (d) Bejan number as a function of solid volume fraction for cases under constant magnetic field and time-periodic magnetic field with various periods where $\omega=\frac{2 \pi}{\tau_{B}}, \tau_{B_{1}}=1, \tau_{B_{2}}=5, \tau_{B_{3}}=10$, and $\mathrm{Ri}=10$.

of metallic nanoparticles. Moreover, it can be seen that the role of external magnetic field is accentuated by using alternating magnetic field with either longer period or larger phase deviation. The cases under an alternating magnetic field with either longer period or larger phase deviation have larger $\overline{\mathrm{Nu}}^{*}$ and smaller $\overline{S_{\text {avg }}} *$ values.

\section{Conclusion}

An accurate finite volume examination has been performed to study the effects of time-periodic magnetic field on MHD mixed convection and the entropy generation of ferrofluid in a linearly heated two-sided cavity. The studied parameters are Richardson number, Hartmann number, nanoparticle volume fraction, period, and phase deviation of alternating magnetic field. The results are represented by streamlines, isotherm contours, time averaged Nusselt number, entropy generation, and Bejan number. From this study, the following conclusions can be drawn:

1. The heat transfer and the entropy generation across the cavity increase and decrease by further increase in Richardson number, respectively;

2. Increasing external magnetic field suppresses fluid intensity of MHD convection of ferrofluid and decreases convective heat transfer within the cavity;

3. Applying time-periodic magnetic field reduces the effect of Lorenz force, whereby the cases under alternating fields have large stream functions in comparison with the cases under constant magnetic fields;

4. One of the possible techniques for entropy generation minimization of unsteady MHD mixed convection inside an enclosed cavity filled by ferrofluid can be applying an external time-periodic magnetic field with short period;

5. The role of alternating magnetic field in both heat transfer reduction and entropy generation enhancement is intensified by adding more volume fraction of ferrite nanoparticles in the ferrofluid. Meanwhile, it is augmented by using a field with either longer period or larger phase deviation;

6. MHD natural convection of ferrofluid inside a two-sided cavity is improved by adding metallic nanoparticles with volume fractions less than $10 \%$, while it is accentuated with further increase in solid volume fraction;

7. The contribution of thermal irreversibility to the 


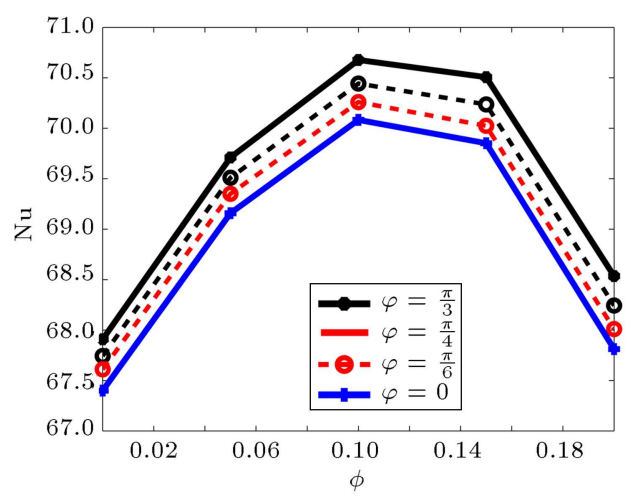

(a)

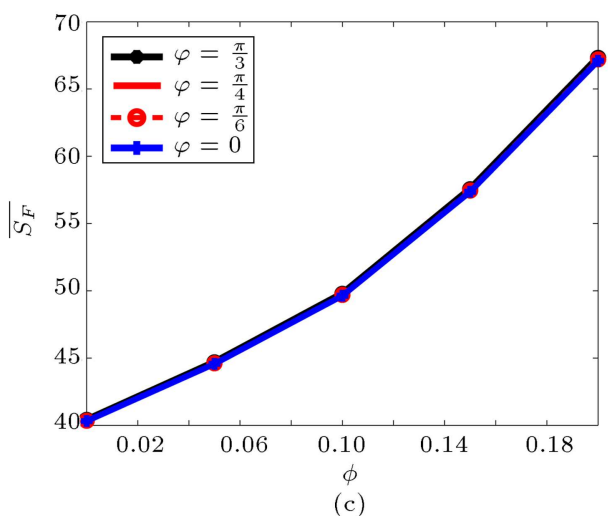

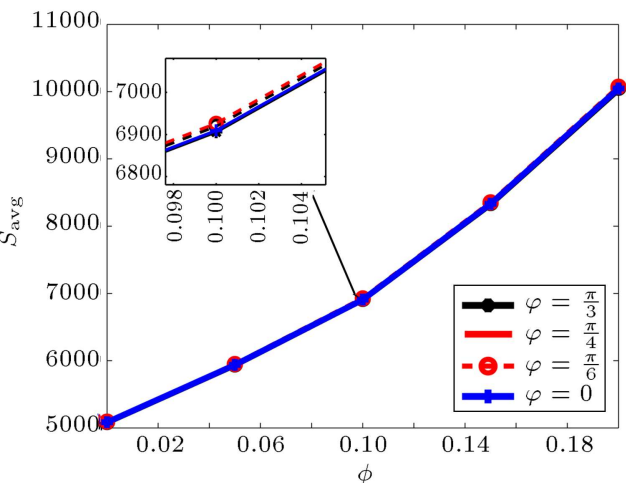

(b)

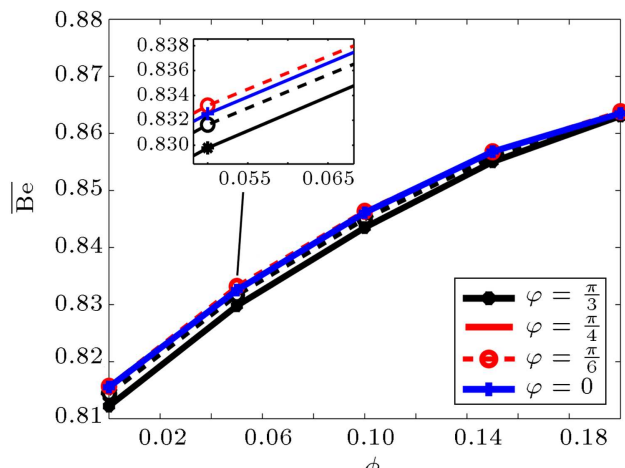

(d)

Figure 12. Variations of average (a) Nusselt number, (b) entropy generation, (c) entropy generation due to fluid friction, and (d) Bejan number as a function of solid volume fraction for cases under periodic magnetic field with amplitude of 25 and different phase deviations when $\mathrm{Ri}=10$.

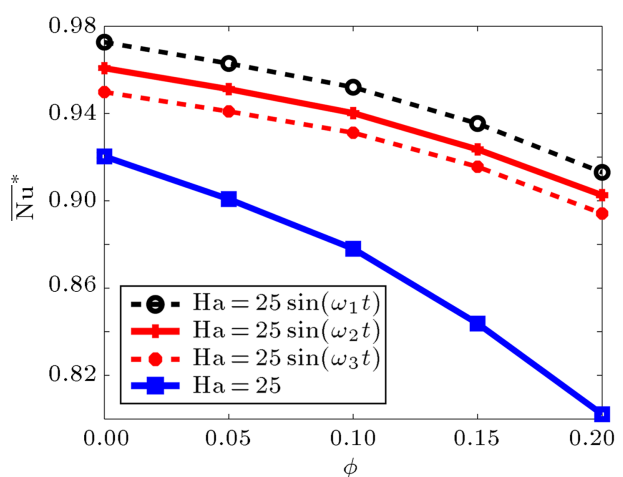

(a)

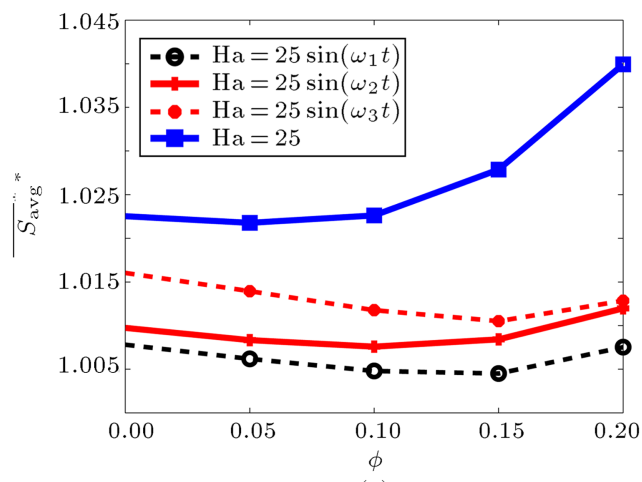

(c)

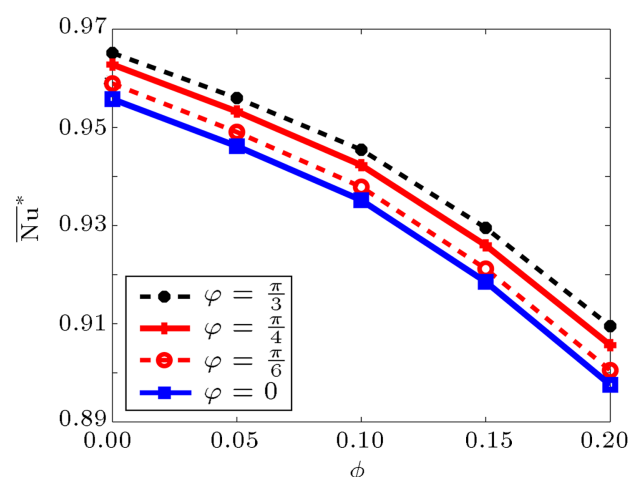

(b)

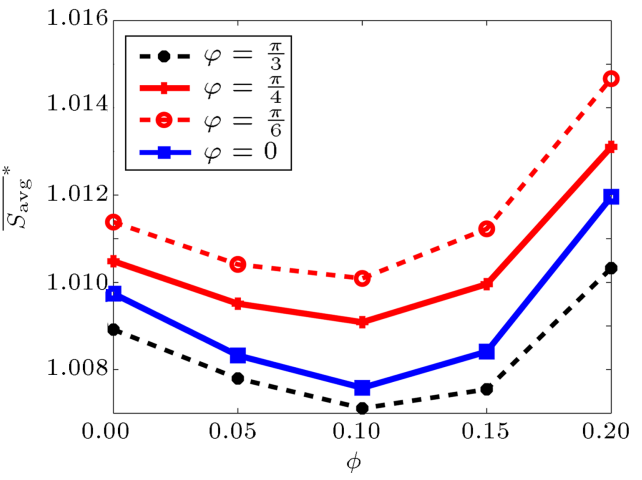

(d)

Figure 13. Variation rates of (a) \& (b) average Nusselt number, and (c) \& (d) average entropy generation, due to applying of external magnetic field as a function of solid volume fraction, where $\omega=\frac{2 \pi}{\tau_{B}}, \tau_{B_{1}}=1, \tau_{B_{2}}=5, \tau_{B_{3}}=10$, and $\mathrm{Ri}=10$. 
total entropy generation is enhanced by further increase in Richardson number and solid volume fraction of ferrite nanoparticles in the ferrofluid.

\section{Nomenclature}

$\overline{\mathrm{Nu}} \quad$ Average Nusselt number

$g \quad$ Acceleration due to gravity $\left(\mathrm{ms}^{-2}\right)$

Gr Grashof number

$L \quad$ Driven cavity width

$\mathrm{Nu} \quad$ Local Nusselt number

$P \quad$ Non-dimensional fluid pressure

$p \quad$ Fluid pressure $\left(\mathrm{Nm}^{-2}\right)$

Pr Prandtl number

Re Reynolds number

Ri Richardson number

$S \quad$ Entropy $\left(\mathrm{JK}^{-1}\right)$

$T \quad$ Fluid temperature (K)

$U, V \quad$ Non-dimensional velocity components

$u, v \quad$ Velocity components $\left(\mathrm{ms}^{-1}\right)$

$V_{0} \quad$ Absolute lid velocity

$X, Y \quad$ Non-dimensional Cartesian coordinate

$x, y \quad$ Cartesian coordinate $(\mathrm{m})$

\section{Greek}

$\alpha$

Thermal diffusivity $\left(\mathrm{m}^{2} \mathrm{~s}\right)$

$\beta$

Thermal expansion coefficient $\left(\mathrm{K}^{-1}\right)$

$\mu$

Dynamic viscosity $\left(\mathrm{kgm}^{-1} \mathrm{~s}^{-1}\right)$

$\nu \quad$ Kinematic viscosity $\left(\mathrm{m}^{2} \mathrm{~s}^{-1}\right)$

$\phi \quad$ Volume fraction of the nanoparticles

$\rho \quad$ Density $\left(\mathrm{kgm}^{-3}\right)$

$\sigma \quad$ Electrical conductivity $\left(\mathrm{Sm}^{-1}\right)$

$\Theta \quad$ Dimensionless temperature

\section{Superscript}

$B \quad$ Magnetic field effect

$F \quad$ Viscous dissipation effect

$h \quad$ High temperature

$l \quad$ Low temperature

$s \quad$ Solid particles

$T \quad$ Heat transfer effect

\section{References}

1. Kefayati, Gh.R. "Natural convection of ferrofluid in a linearly heated cavity utilizing LBM", J. Molecular Liquid, 191, pp. 1-9 (2014).
2. Kefayati, Gh.R. "Mesoscopic simulation of mixed convection on non-Newtonian nanofluids in a two sided lid-driven enclosure", Powder Technol., 26, pp. 576588 (2015).

3. Nemati, H., Farhadi, M., Sedighi, K., Ashorynejad, H.R. and Fattahi, E. "Magnetic field effects on natural convection flow of nanofluid in a rectangular cavity using the Lattice Boltzmann model", Scientia Iranica, 19, pp. 303-310 (2012).

4. Kefayati, Gh.R. "Lattice Boltzmann simulation of natural convection in a nanofluid-filled inclined square cavity at presence of magnetic field", Scientia Iranica, 20, pp. 1517-1527 (2013).

5. Choi, S.U.S. "Enhancing thermal conductivity of fluid with nanoparticles", ASME Fluid Eng. Div., 23, pp. 99-105 (1995).

6. Selimefendigil, F., Oztop, H.F. and Al-Salem, Kh. "Natural convection of ferrofluids in partially heated square enclosures", J. Mag. Mag. Mat., 372, pp. 122133 (2014).

7. Nanjundappa, C.E., Shivakumara, I.S. and Prakash, H.N. "Effects of Coriolis force on thermomagnetic convection in a ferrofluid saturating porous medium: A weakly nonlinear stability analysis", J. Mag. Mag. Mat., 370, pp. 140-149 (2014).

8. Rahman, M.M., Mojumder. S., Saha, S., Joarder, A.H., Saidur, R. and Naim, A.G. "Numerical and statistical analysis on unsteady magnetohydrodynaimc convection in a semi-circular enclosure filled with ferrofluid", Int. J. Heat Mass Transfer, 89, pp. 13161330 (2015).

9. Mohammadpourfard, M. "Numerical study of ferrofluid flow and heat transfer in the presence of a nonuniform magnetic field in rectangular microchannel", Heat Transfer-Asian Res., 41, pp. 302-317 (2012).

10. Sheikholeslami, M. and Ganji, D.D. "Ferrohydrodynamic and magnetohydrodynamic effects on ferrofluid flow and convective heat transfer", Energy, 75, pp. 400-410 (2014).

11. Selimefendigil, F., Oztop, H.F. and Chamkha, A.J. "MHD mixed convection and entropy generation of nanofluid filled lid driven cavity under the influence of inclined magnetic fields imposed to its upper and lower diagonal triangular domains", J. Mag. Mag. Mat., 406, pp. 266-281 (2016).

12. Ahrar, A.J. and Djavareshkian, M.H. "Lattice Boltzmann simulation of a $\mathrm{Cu}$-water nanofluid filled cavity in order to investigate the influence of volume fraction and magnetic field specifications on flow and heat transfer", J. Molecular Liquid, 215, pp. 328-338 (2016).

13. Rashidi, M.M., Nasiri, M., Khezerloo, M. and Laraqi, N. "Numerical investigation of magnetic field effect on mixed convection heat transfer of nanofluid in a channel with sinusoidal walls", J. Mag. Mag. Mat., 401, pp. 159-168 (2016). 
14. Garosi, F. and Hosseininejad, F. "Numerical study of natural and mixed convection heat transfer between differentially heated cylinders in an adiabatic enclosure filled with nanofluid", J. Molecular Liquid, 216, pp. 117 (2016).

15. Hamilton, R.I. and Crosser, O.K. "Thermal conductivity of heterogeneous two-component system", Ind. Eng. Chem. Fundam., 1, pp. 187-191 (1962).

16. Moumni, H., Welhezi, H., Djebali, R. and Sediki, E. "Accurate finite volume investigation of nanofluid mixed convection in two-sided lid-driven cavity including discrete sources", Appl. Math. Model., 39, pp. 4164-4179 (2015).

17. Sadrhosseini, H., Sehat, A. and Shafii, M.B. "Effect of magnetic field on internal forced convection of ferrofluid flow in porous media", Exp. Heat Transfer, 29, pp. 1-16 (2016).

18. Nayak, R.K., Bhattacharyya, S. and Pop, I. "Numerical study on mixed convection and entropy generation of $C u$-water nanofluid in a differentially heated skewed enclosure", Int. J. Heat Mass Transfer, 85, pp. 620-634 (2015).

19. Bejan, A. "A study of entropy generation in fundamental convection heat transfer", ASME J. Heat Transfer, 101, pp. 718-725 (1979).

20. Mejri, I. and Mahmoudi, A. "MHD natural convection in a nanofluid-filled open enclosure with a sinusoidal boundary condition", Chem. Eng. Res. Des., 98, pp. 1-16 (2015).

21. Sarris, I.E., Zikos, G.K., Grecos, A.P. and Vlachos, N.S. "On the limits of validity of the low magnetic Reynolds number approximation in MHD naturalconvection heat transfer", Numerical Heat Transfer, Part B: Fundamentals: An International Journal of Computation and Methodology, 50, pp. 157-180 (2006).

22. Mehrez, Z., Cafsi, A.E., Belghith, A. and Le Quere, P. "The entropy generation analysis in the mixed convection assisting flow of $\mathrm{Cu}$-water nanofluid in an inclined open cavity", Adv. Powder Technol., 26, pp. 1442-1451 (2015).

23. Chorin, A.J. "Numerical simulation of the NavierStokes equations", Math. Comput., 22, pp. 745-762 (1968).

24. Chorin, A.J. "On the convergence of discrete approximations to the Navier-Stokes equations", Math. Comput., 23, pp. 341-353 (1969).

25. Hayase, T., Humphery, J.A.C. and Greif, R. "A consistently formulated QUICK scheme for fast and stable convergence using finite volume iterative calculation procedure", J. Comput. Phys., 98, pp. 108-118 (1992).

26. Davis, I.A. and Hager, W.W. "Modifying a sparse Cholesky factorization", SIAM J. Matrix Anal. Appl., 20, pp. 606-627 (1999).
27. Rahman, M.M., Alim, M.A. and Sarker, M.M.A. "Numerical study on conjugate effect of joule heating and magneto-hydrodynamics mixed convection in an obstructed lid-driven square cavity", Int. J. Heat Mass Transfer, 37, pp. 524-534 (2010).

28. Al-Salem, Kh., Oztop, H.F., Pop, I. and Varol, Y. "Effects of mixing lid direction on MHD mixed convection in a linearly heated cavity", Int. J. Heat Mass Transfer, 55, pp. 1103-1112 (2012).

29. Dixit, H.N. and Babu, V. "Simulation of high Rayleigh number natural convection in a square cavity using the lattice Boltzmann method", Int. J. Heat Mass Transfer, 49, pp. 727-739 (2006).

30. Kuznik, F., Vareilles, J., Rusaouen, G. and Krauss, G. "A double-population lattice Boltzmann method with non-uniform mesh for the simulation of natural convection in a square cavity", Int. J. Heat Fluid Flow, 28, pp. 862-870 (2007).

31. Pekmen, B. and Tezer-Sezgin, M. "MHD flow and heat transfer in a lid-driven porous enclosure", Computers and Fluids, 89, pp. 191-199 (2013).

32. El-Shehabey, H.M. and Ahmed, S.E. "MHD mixed convection in a lid-driven cavity filled by a nanofluid with sinusoidal temperature distribution on the both vertical walls using Buongiorno's nanofluid model", Int. J. Heat Mass Transfer, 88, pp. 181-202 (2015).

33. Sheremet, M.A. and Pop, I. "Mixed convection in a liddriven square cavity filled by a nanofluid: Buongiorno's mathematical model", Appl. Math. Comput., 266, pp. $792-808$ (2015).

34. Mahmoud, A., Mejri, I., Abbasi, M.A. and Omri, A. "Analysis of MHD natural convection in a nanofluidfilled open cavity with non-uniform boundary condition in the presence of uniform heat generation/absorption", Powder Technol., 269, pp. 275-289 (2015).

35. Nanna, A.G. "Experimental model of temperature driven nanofluid", Trans. ASME J. Heat Transfer, 129, pp. 697-704 (2007).

\section{Biography}

Omid Ghaffarpasand received his $\mathrm{PhD}$ from Department of Physics, University of Isfahan, Iran, in 2012, where he is currently a full-time member. He is also the head of air pollution and solid waste matter research group of environmental research institute, University of Isfahan, Iran. His research fields include fluid dynamics, computational fluid dynamics, aerosol technology, aerosol propagation and deposition mechanisms, heat transfer, and fluid flow of Magnetohydrodynamic convection. 